Supporting information for

\title{
Synthesis of Bisubstrate and Donor Analogs of Sialyltransferase and Their Inhibitory Activities
}

Masayuki Izumi,* Katsuhiro Wada, Hideya Yuasa, and Hironobu Hashimoto

Table of contents

$\begin{array}{ll}\text { General procedures } & \mathrm{S} 2\end{array}$

${ }^{1} \mathrm{H}$ and ${ }^{13} \mathrm{C}$ NMR spectra of $\mathbf{1 0} \quad \mathrm{S} 3,4$

${ }^{1} \mathrm{H}$ and ${ }^{13} \mathrm{C}$ NMR spectra of $\mathbf{1 2}$ S5,6

${ }^{1} \mathrm{H}$ and ${ }^{13} \mathrm{C}$ NMR spectra of $\mathbf{1 3} \quad$ S7,8

$\begin{array}{lr}{ }^{1} \mathrm{H} \text { and }{ }^{13} \mathrm{C} \text { NMR spectra of } \mathbf{1 4} & \mathrm{S} 9.10\end{array}$

${ }^{1} \mathrm{H}$ and ${ }^{13} \mathrm{C}$ NMR spectra of $\mathbf{1 5} \quad \mathrm{S} 11,12$

${ }^{1} \mathrm{H}$ and ${ }^{13} \mathrm{C}$ NMR spectra of $\mathbf{1 6} \quad \mathrm{S} 13,14$

${ }^{1} \mathrm{H}$ and ${ }^{13} \mathrm{C}$ NMR spectra of $\mathbf{1 7} \quad \mathrm{S} 15,16$

$\begin{array}{lr}{ }^{1} \mathrm{H} \text { and }{ }^{13} \mathrm{C} \text { NMR spectra of } \mathbf{1 8} & \mathrm{S} 17,17\end{array}$

${ }^{1} \mathrm{H}$ and ${ }^{13} \mathrm{C}$ NMR spectra of $\mathbf{1 9} \quad \mathrm{S} 19,20$

${ }^{1} \mathrm{H}$ and ${ }^{13} \mathrm{C}$ NMR spectra of $\mathbf{2 1} \quad$ S21,22

${ }^{1} \mathrm{H}$ and ${ }^{13} \mathrm{C}$ NMR spectra of $4 \quad$ S23,24

${ }^{1} \mathrm{H}$ and ${ }^{13} \mathrm{C}$ NMR spectra of $5 \quad$ S25,26

${ }^{1} \mathrm{H}$ and ${ }^{13} \mathrm{C}$ NMR spectra of $\mathbf{2 2} \quad$ S27,28

$\begin{array}{ll}{ }^{1} \mathrm{H} \text { NMR spectrum of } \mathbf{2 3} & \text { S29 }\end{array}$

${ }^{1} \mathrm{H}$ and ${ }^{13} \mathrm{C}$ NMR spectra of $6 \quad$ S30,31

${ }^{1} \mathrm{H}$ NMR spectrum of $\mathbf{2 4} \quad$ S32

$\begin{array}{ll}{ }^{1} \mathrm{H} \text { and }{ }^{13} \mathrm{C} \text { NMR spectra of } 7 & \text { S33,34 }\end{array}$

$\begin{array}{ll}{ }^{1} \mathrm{H} \text { NMR spectrum of } \mathbf{2 6} & \mathbf{S 3 5}\end{array}$

$\begin{array}{ll}{ }^{1} \mathrm{H} \text { and }{ }^{13} \mathrm{C} \text { NMR spectra of } 8 & \text { S36,37 }\end{array}$ 
General procedures. ${ }^{1} \mathrm{H}$ NMR spectra were recorded at 270 or $400 \mathrm{MHz} .{ }^{13} \mathrm{C}$ NMR spectra were recorded at 67.8 or $100 \mathrm{MHz}$, and ${ }^{31} \mathrm{P}$ NMR spectra were recorded at $109 \mathrm{MHz}$. Where indicated, NMR peak assignments were made using COSY; all others are subjective. All chemical shifts are quoted on the $\delta$-scale and were referenced to tetramethylsilane $\left(\delta=0\right.$ in $\left.\mathrm{CDCl}_{3}\right)$ or $\mathrm{HDO}(\delta=4.80$ in $\left.\mathrm{D}_{2} \mathrm{O}\right)$ for ${ }^{1} \mathrm{H} \mathrm{NMR}$, and $\mathrm{CDCl}_{3}\left(\delta=77.0\right.$ in $\left.\mathrm{CDCl}_{3}\right)$ or NAc $\left(\delta=23.1\right.$ in $\left.\mathrm{D}_{2} \mathrm{O}\right)$ for ${ }^{13} \mathrm{C}$ NMR as an internal standard, and $85 \% \mathrm{H}_{3} \mathrm{PO}_{4}(\delta=0)$ for ${ }^{31} \mathrm{P}$ NMR as an external standard. Combinations of the following abbreviations are used to describe NMR spectra: s, singlet; $d$, doublet; $t$, triplet; q, quartet; qu, quintet, m, multiplet; br, broad. Low and high-resolution mass spectra were recorded using electrospray ionization techniques with time-of-flight detector. Thin layer chromatography (TLC) was carried out on glass plates coated with silica gel 60F254 (E. Merck). Plates were visualized by irradiation with UV lamp, or dipping in $1 \% \mathrm{Ce}\left(\mathrm{SO}_{4}\right)_{2}-1.5 \%\left(\mathrm{NH}_{4}\right)_{6} \mathrm{Mo}_{7} \mathrm{O}_{24}-10 \% \mathrm{H}_{2} \mathrm{SO}_{4}$, or orcinol in $10 \% \mathrm{H}_{2} \mathrm{SO}_{4}-\mathrm{EtOH}$ and charred. Silica gel column chromatography was performed using Wakogel C300 (40-75 $\mu \mathrm{m}$, Wako Chemical). Spherical silica gel 60N (0.1-0.2 mm, neutral) was purchased from Kanto Kagaku. Rat recombinant $\alpha-2,6$-sialyltransferase, $\alpha-2,3-(N)$-sialyltransferase and CMPNeu5Ac were purchased from Calbiochem. Inertsil-ODS-3 (5 $\mu \mathrm{m}, \phi 4.6 \mathrm{~mm} \times 100 \mathrm{~mm})$ HPLC column was purchased from GL Science. Dehydrated THF was purchased from Kanto Kagaku. Dehydrated toluene was purchased from Wako Chemical. 
$270 \mathrm{MHz}{ }^{1} \mathrm{H}$ NMR spectrum of compound $\mathbf{1 0}$

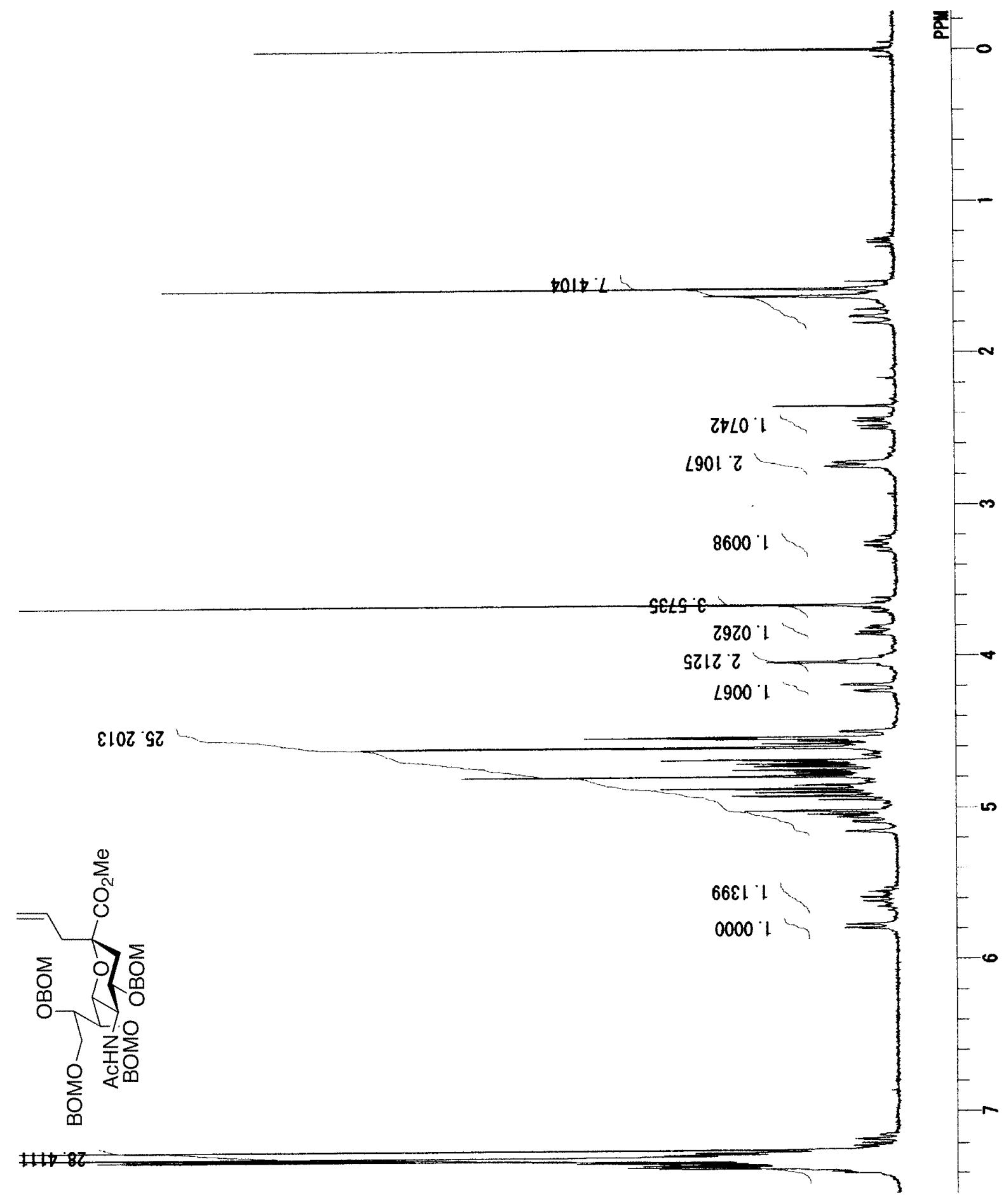


$67.8 \mathrm{MHz}{ }^{13} \mathrm{C}$ NMR spectrum of compound $\mathbf{1 0}$

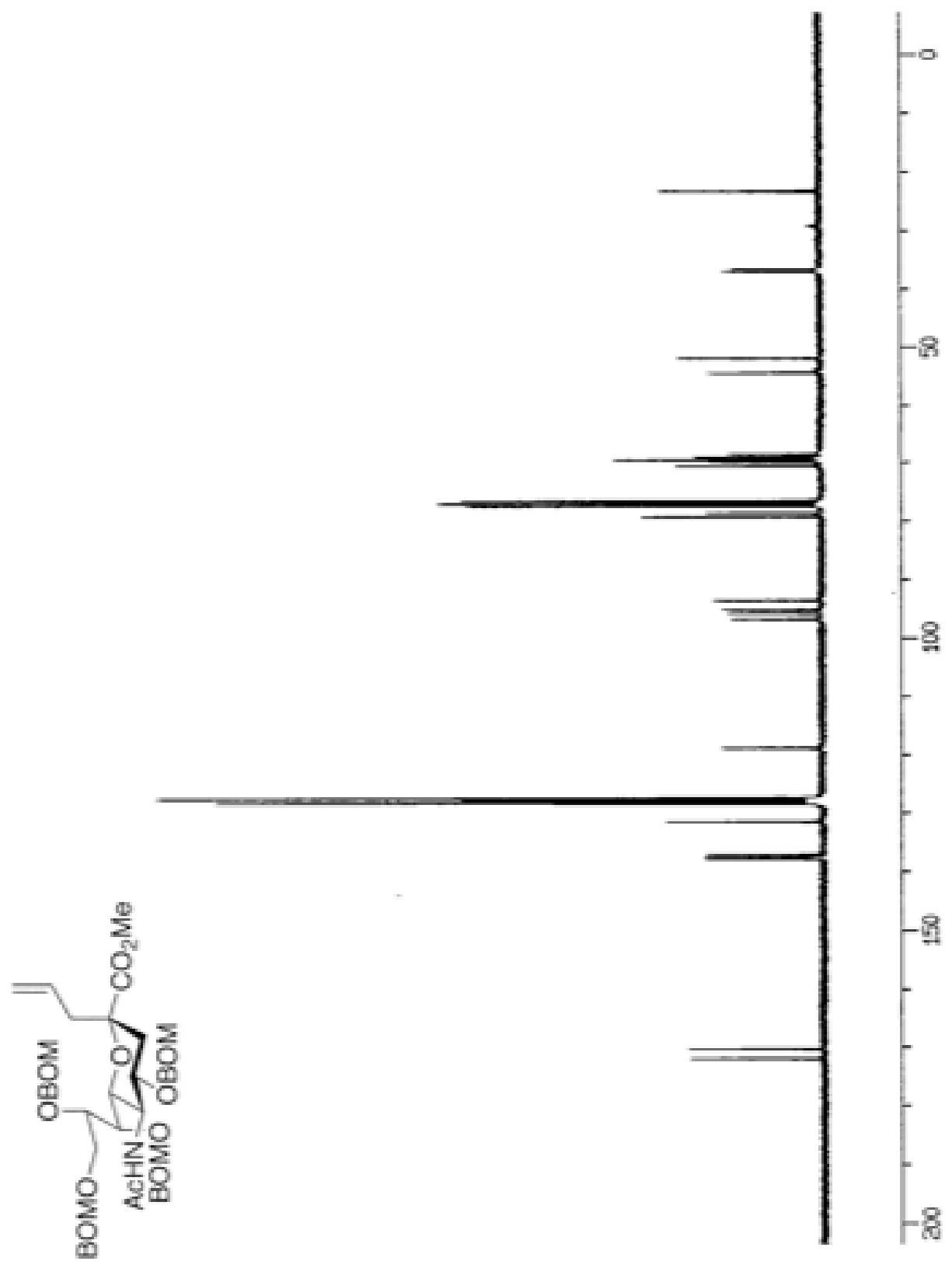


$270 \mathrm{MHz}{ }^{1} \mathrm{H}$ NMR spectrum of compound 12

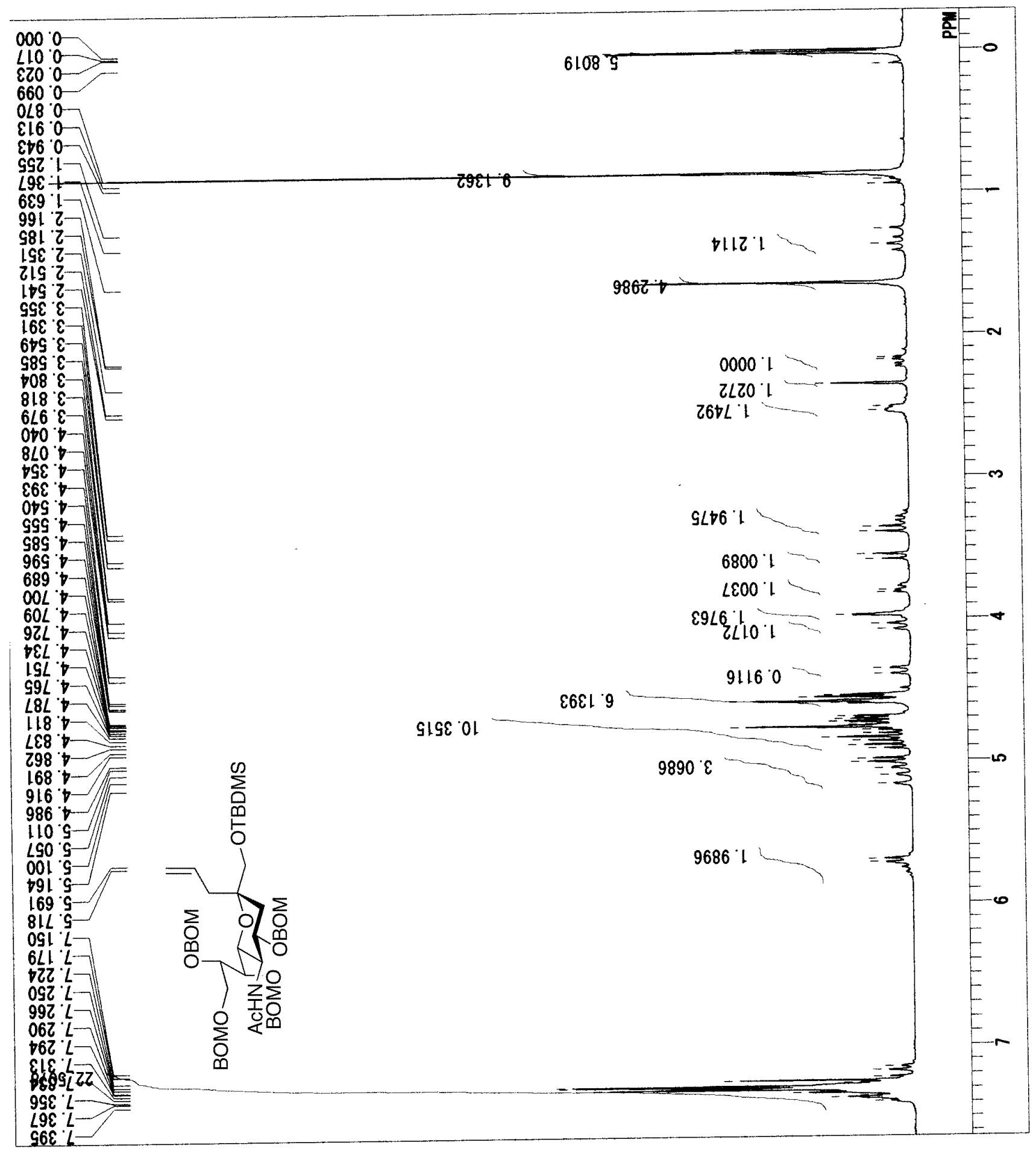


$67.8 \mathrm{MHz}{ }^{13} \mathrm{C}$ NMR spectrum of compound 12

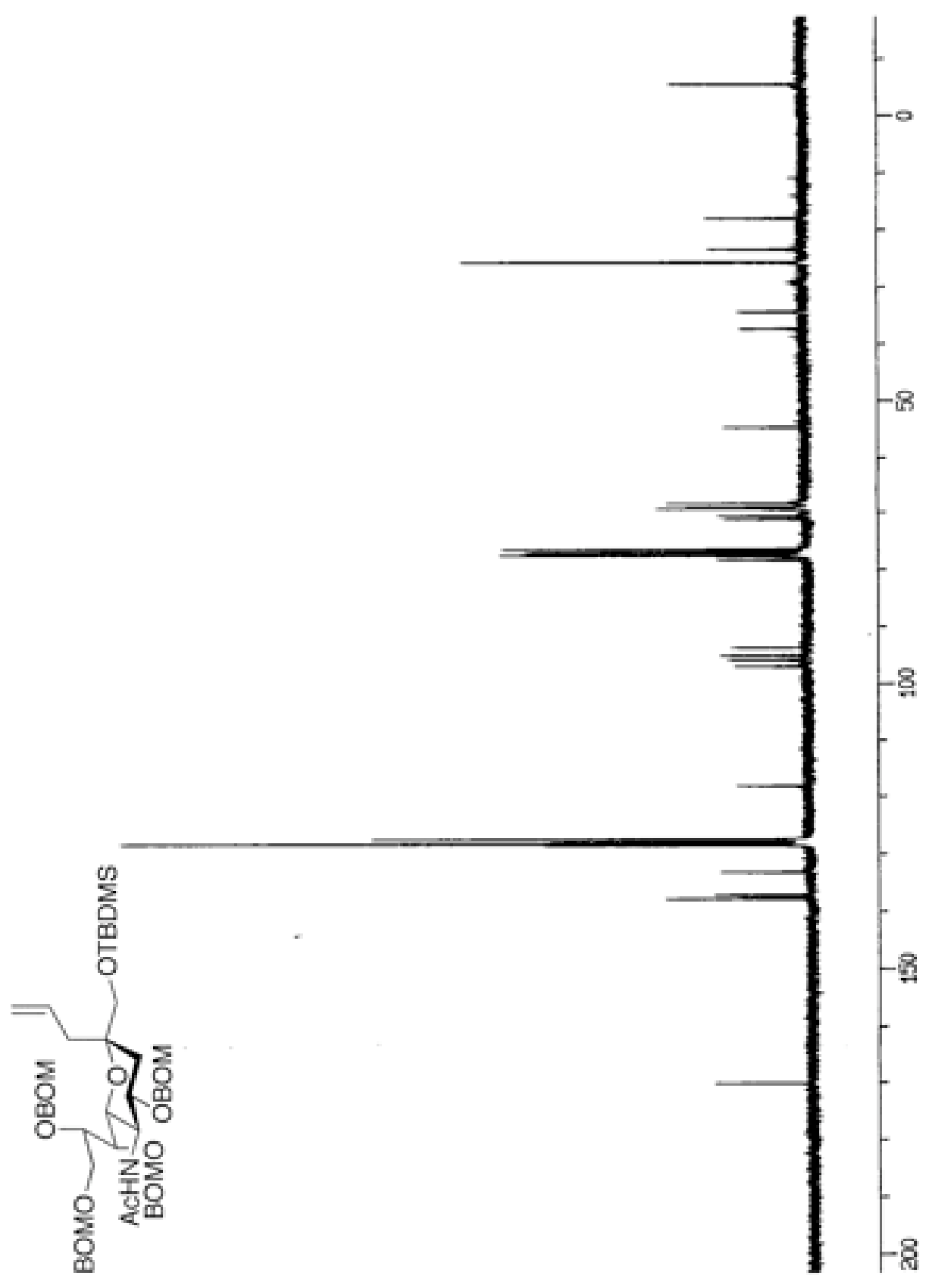


$270 \mathrm{MHz}{ }^{1} \mathrm{H}$ NMR spectrum of compound $\mathbf{1 3}$

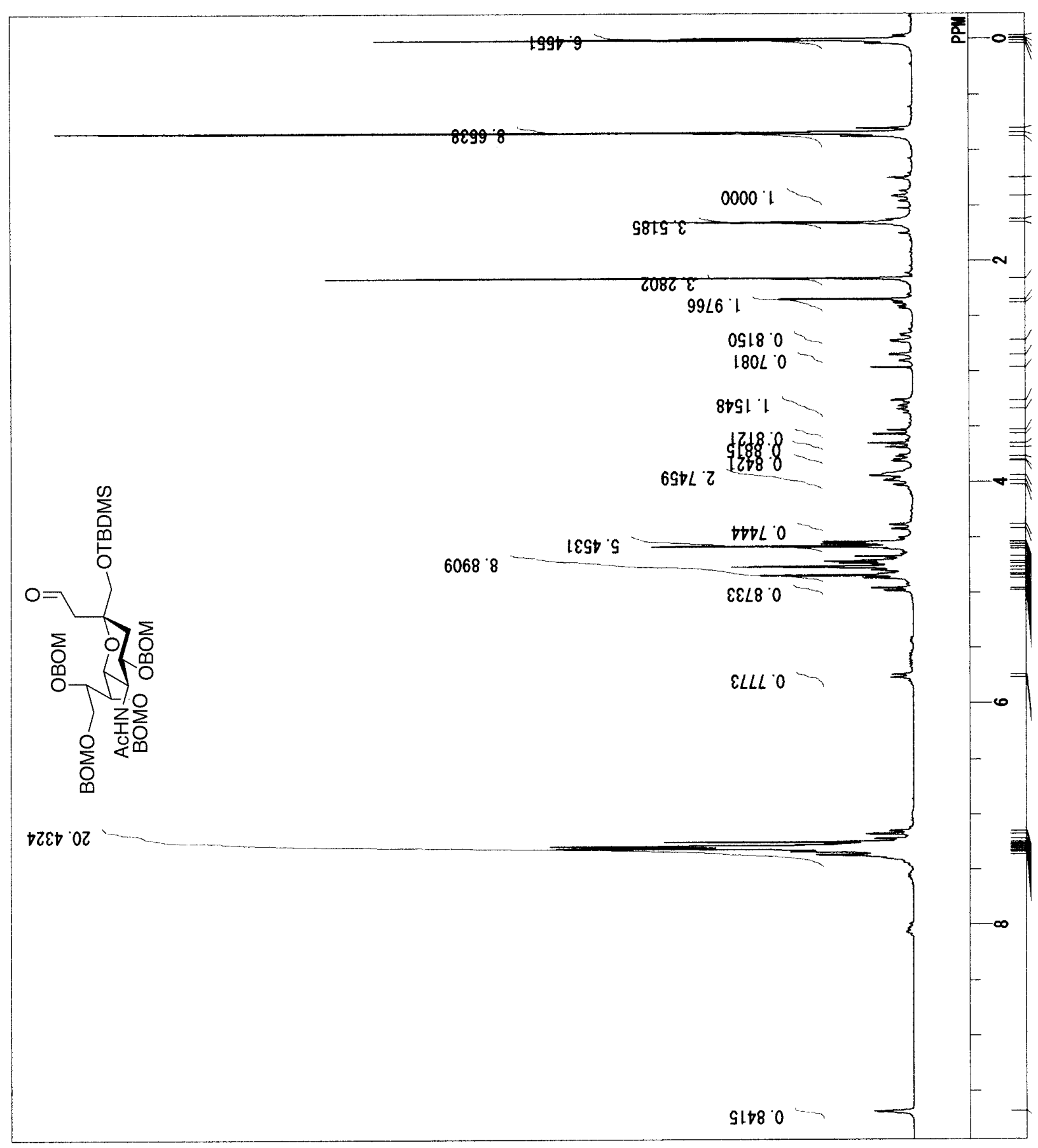




\section{$67.8 \mathrm{MHz}{ }^{13} \mathrm{C}$ NMR spectrum of compound $\mathbf{1 3}$}

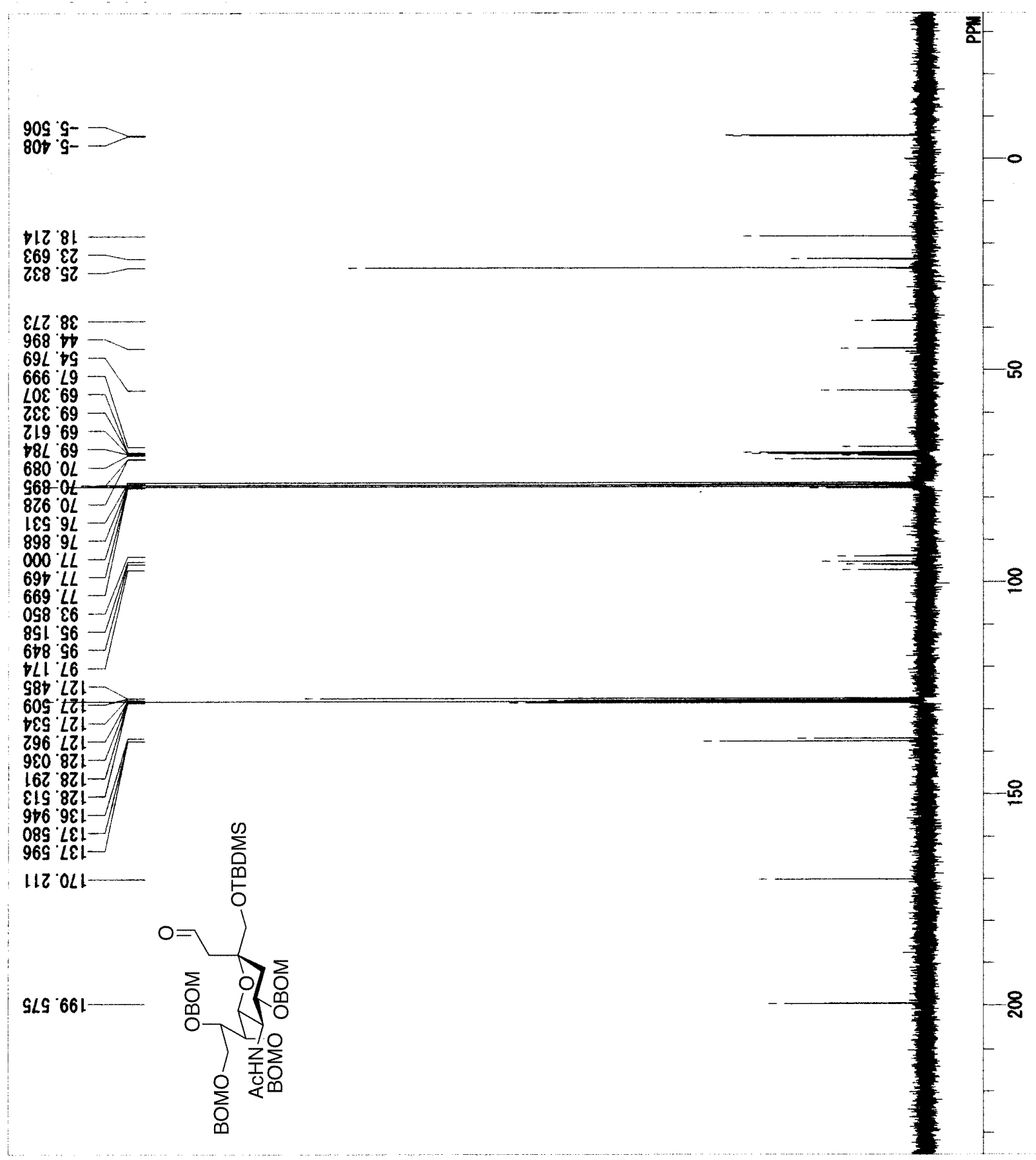


$270 \mathrm{MHz}{ }^{1} \mathrm{H}$ NMR spectrum of compound 14

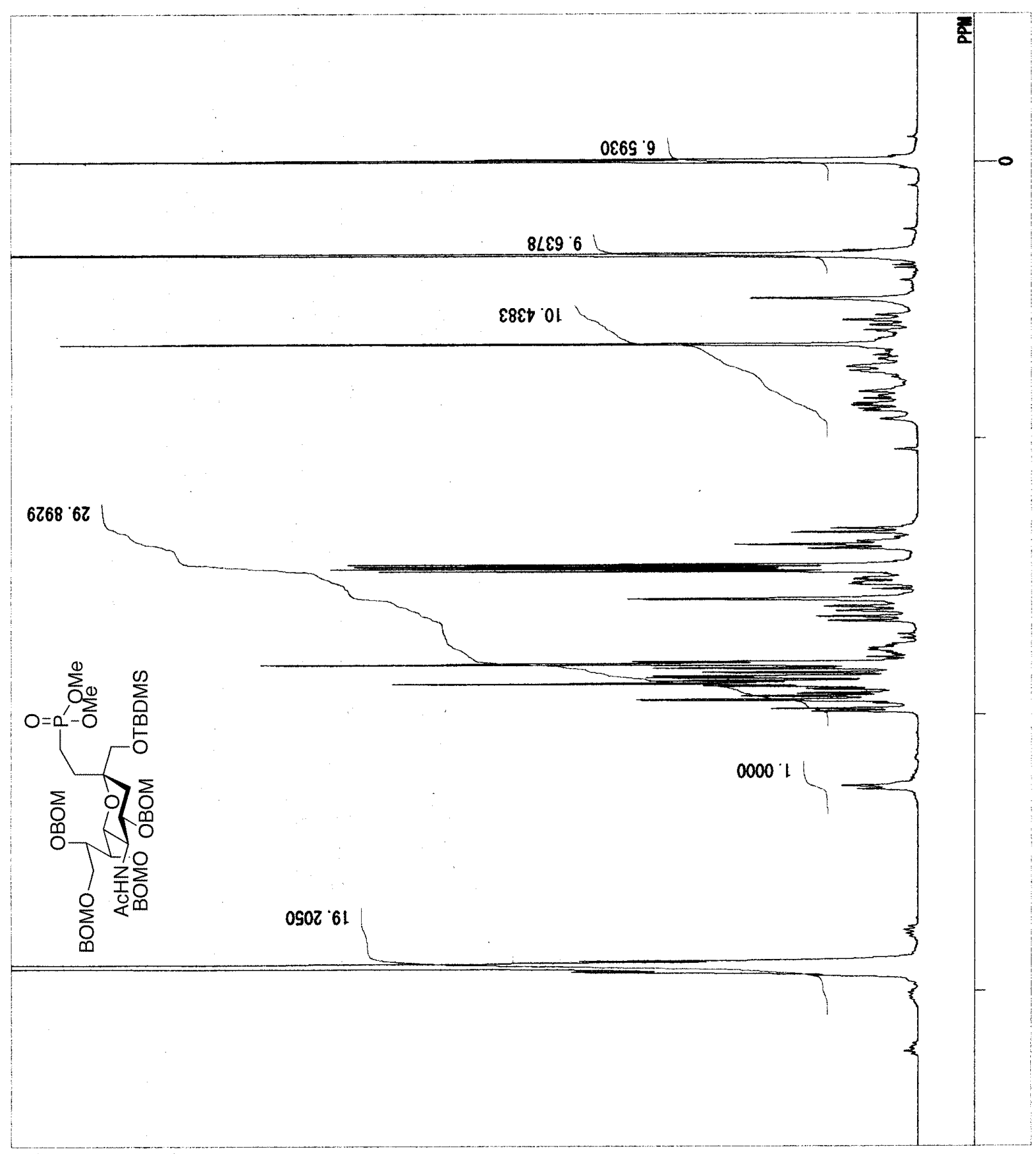


$67.8 \mathrm{MHz}{ }^{13} \mathrm{C}$ NMR spectrum of compound $\mathbf{1 4}$

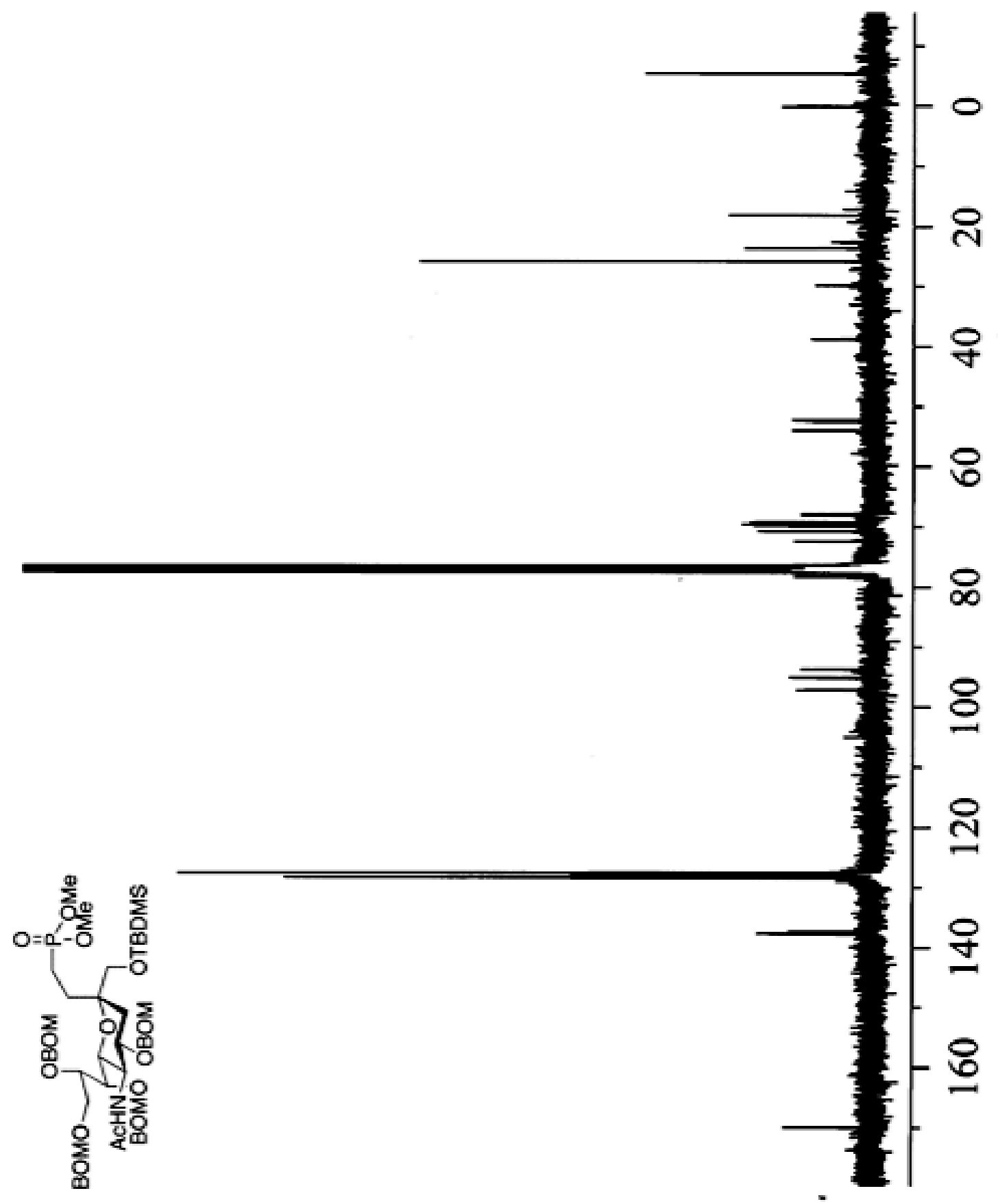


$270 \mathrm{MHz}{ }^{1} \mathrm{H}$ NMR spectrum of compound 15

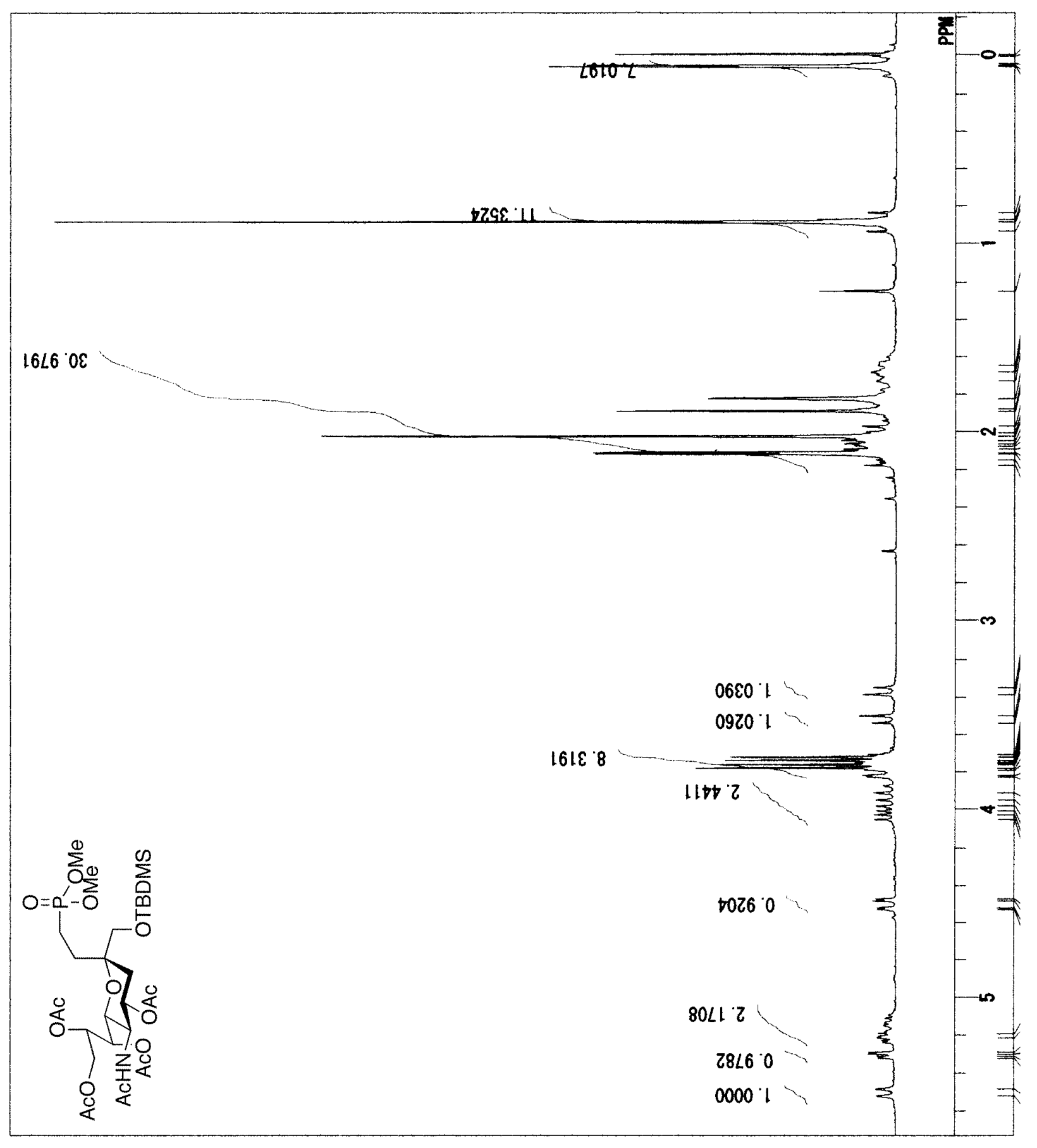


$67.8 \mathrm{MHz}{ }^{13} \mathrm{C}$ NMR spectrum of compound $\mathbf{1 5}$

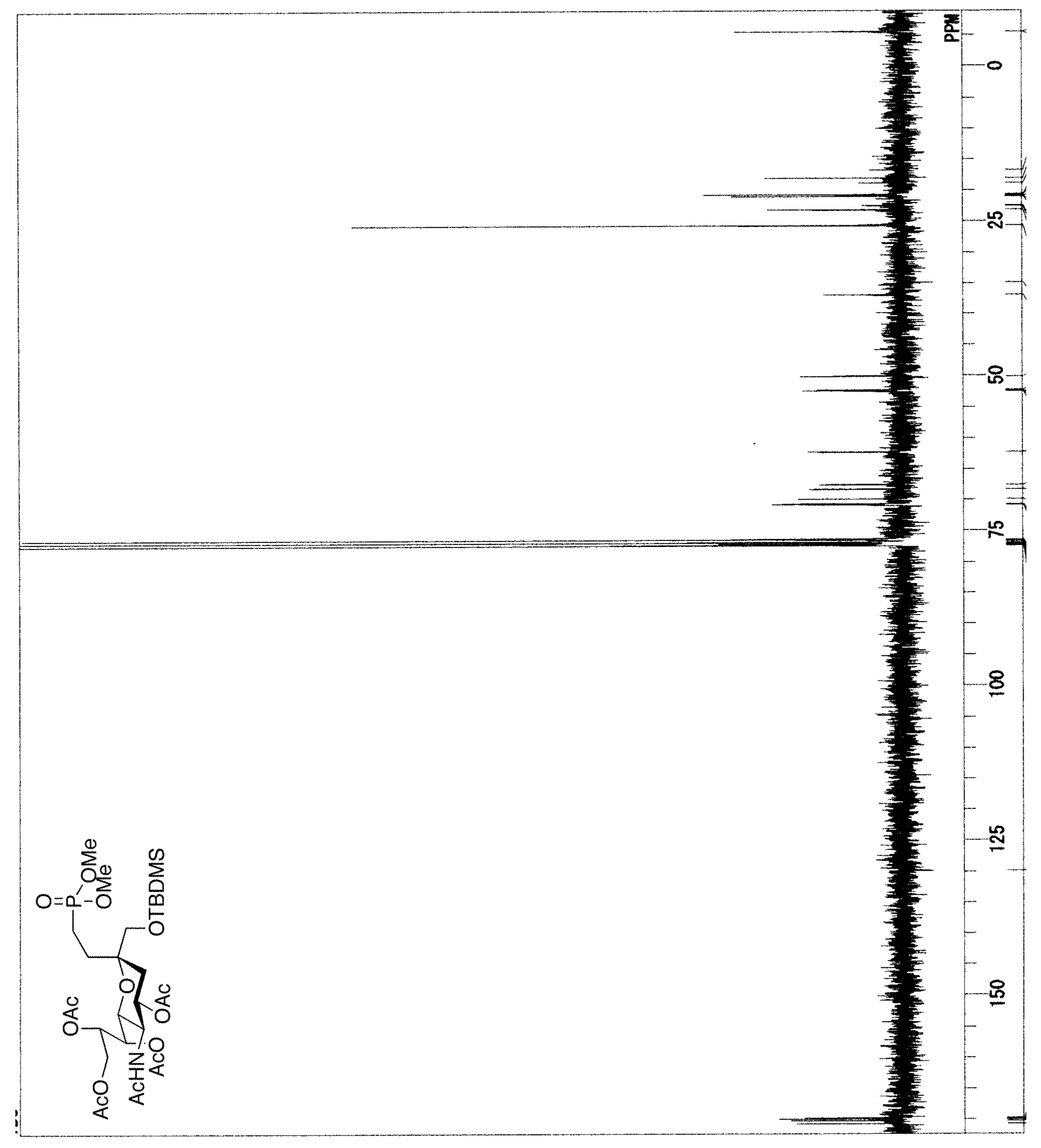


$270 \mathrm{MHz}{ }^{1} \mathrm{H}$ NMR spectrum of compound $\mathbf{1 6}$

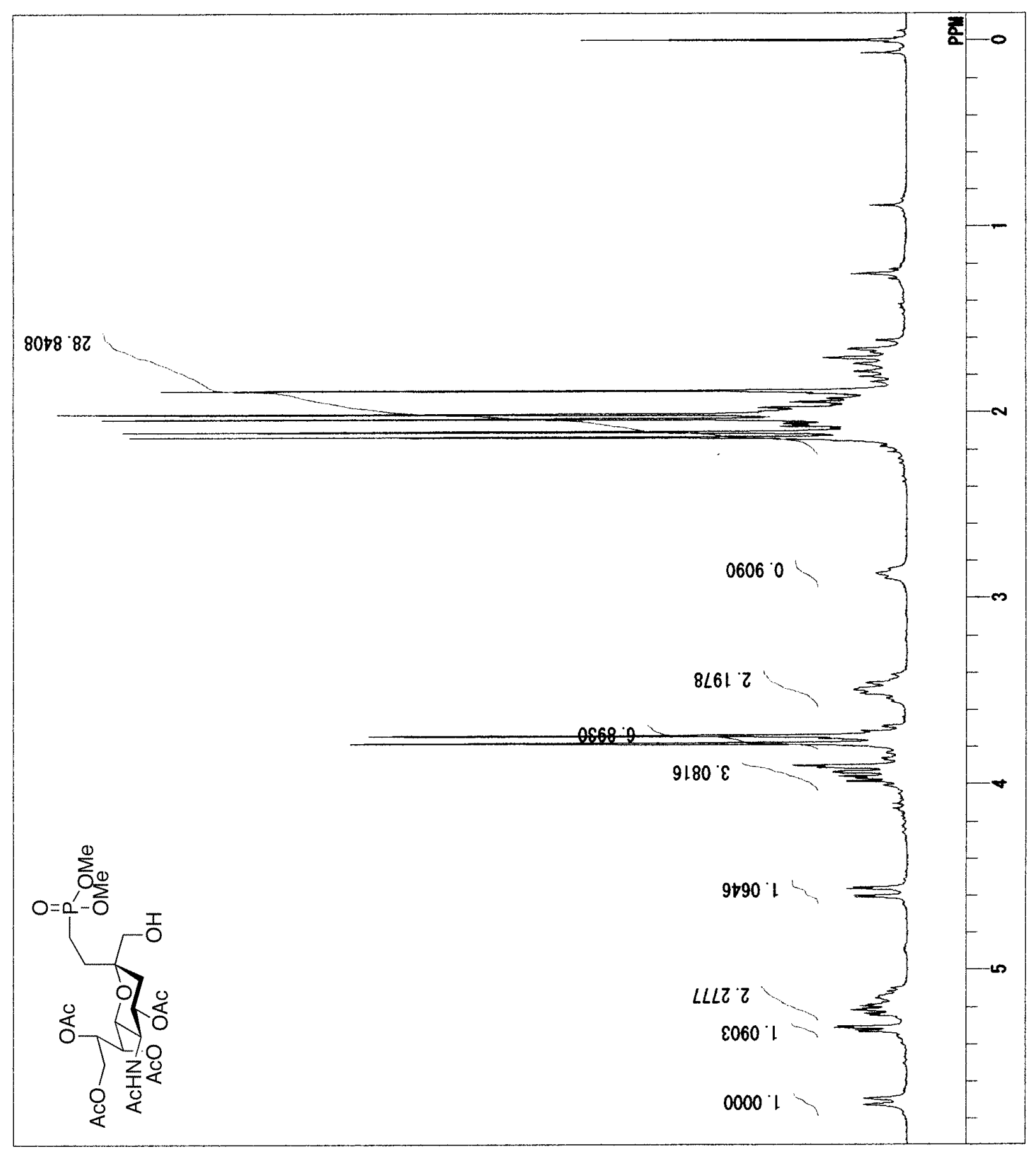


$67.8 \mathrm{MHz}{ }^{13} \mathrm{C}$ NMR spectrum of compound $\mathbf{1 6}$

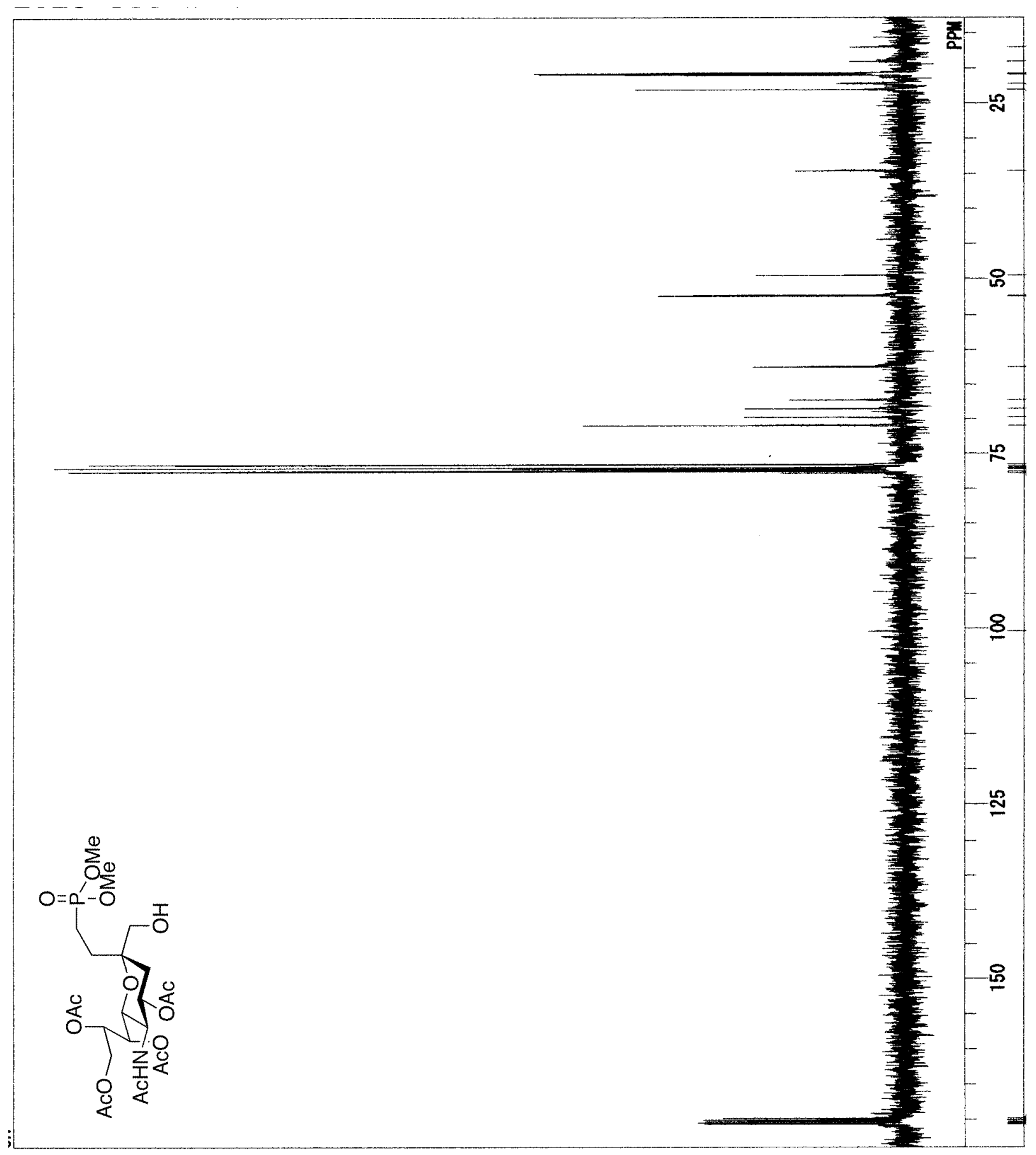


$270 \mathrm{MHz}{ }^{1} \mathrm{H}$ NMR spectrum of compound $\mathbf{1 7}$

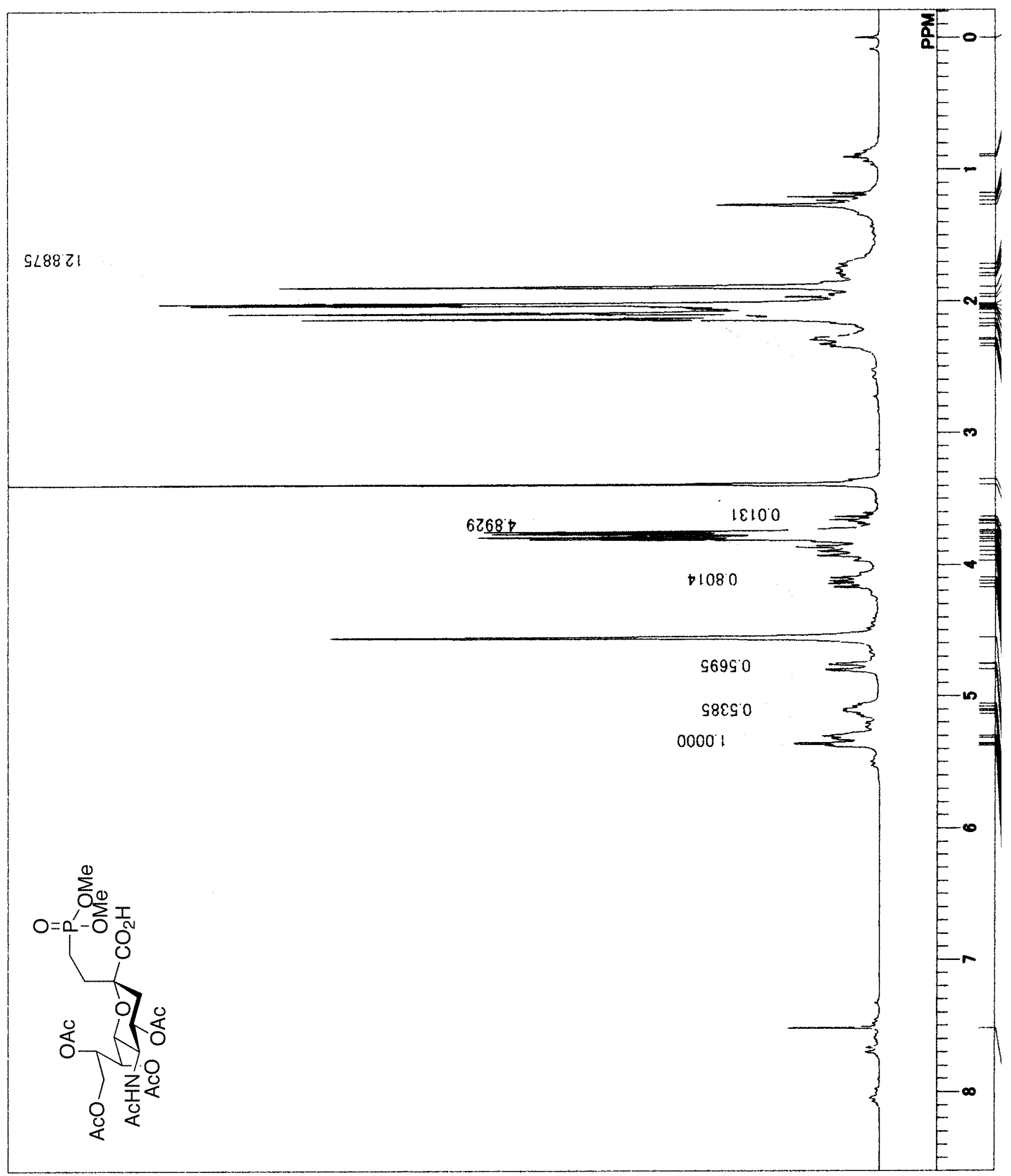


67.8 $\mathrm{MHz}{ }^{13} \mathrm{C}$ NMR spectrum of compound 17

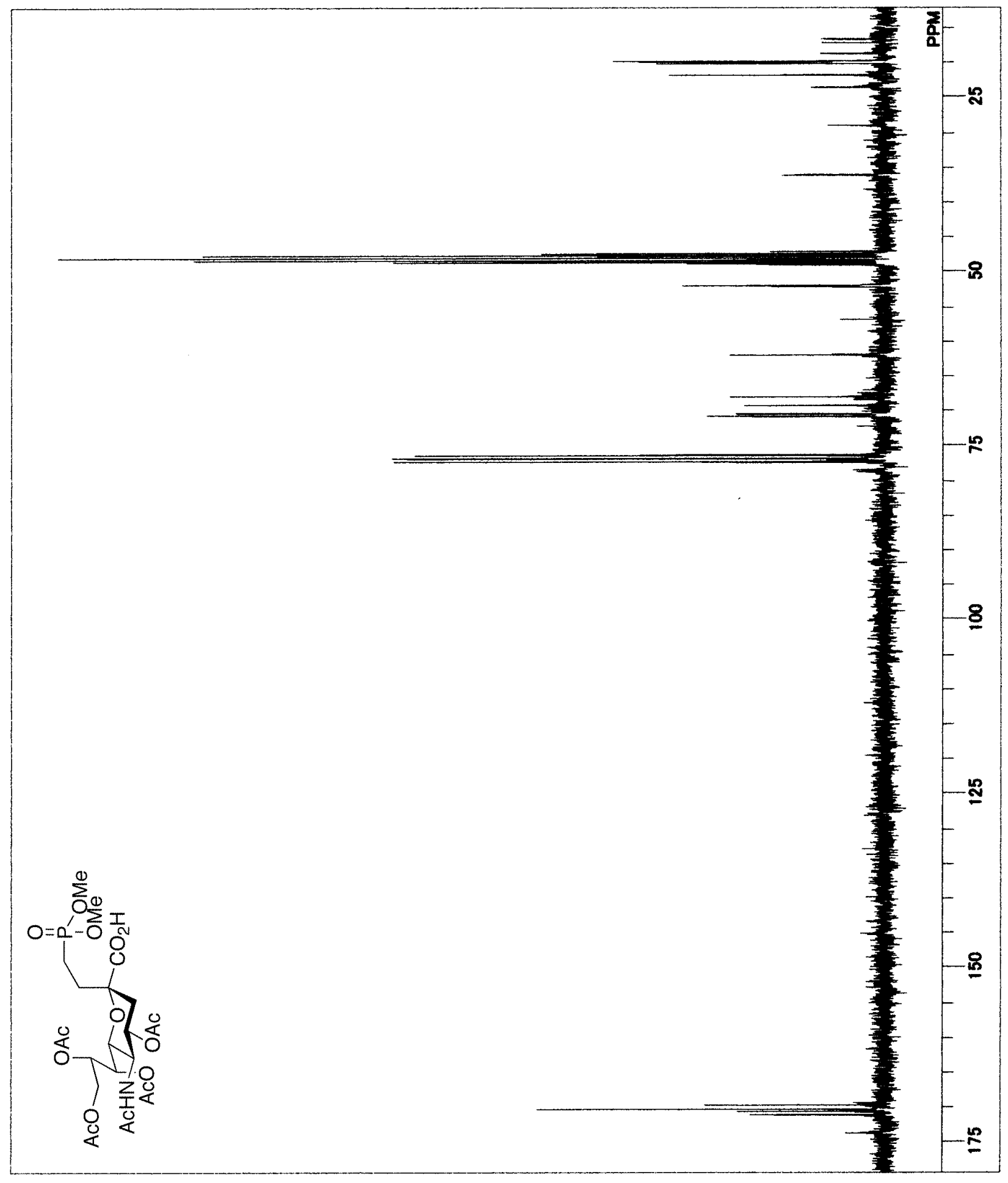


$270 \mathrm{MHz}{ }^{1} \mathrm{H}$ NMR spectrum of compound 18

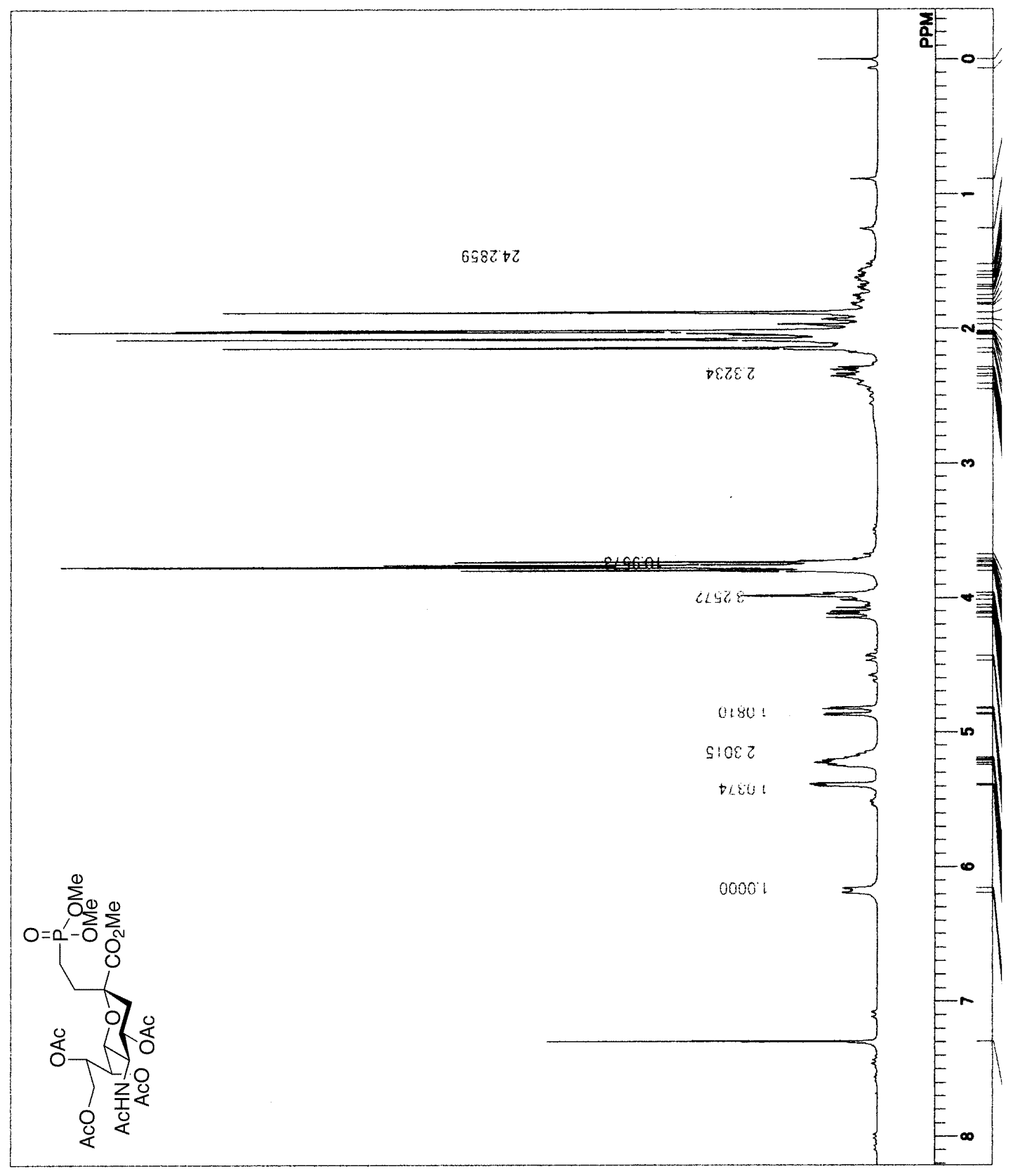


$67.8 \mathrm{MHz}{ }^{13} \mathrm{C}$ NMR spectrum of compound $\mathbf{1 8}$

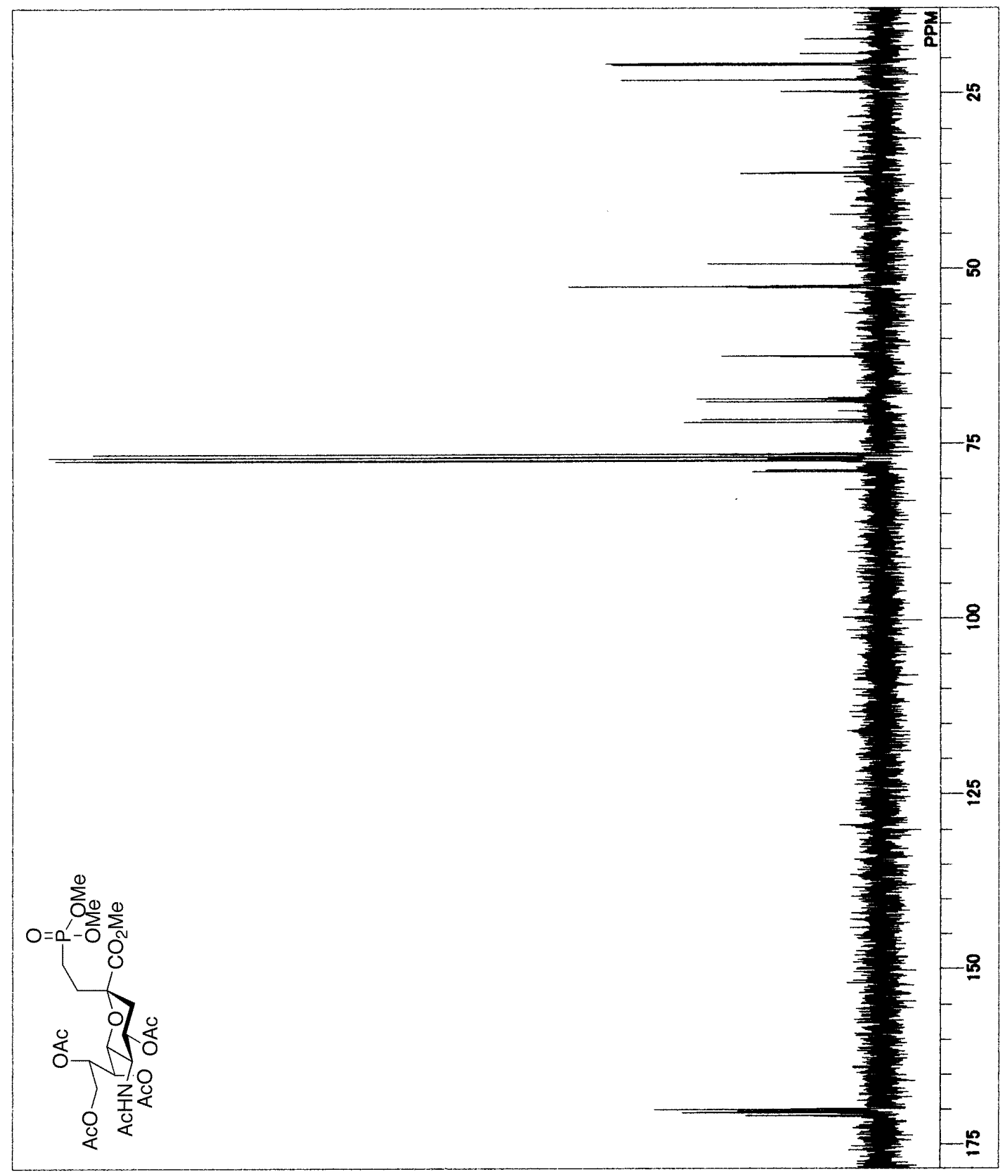


$270 \mathrm{MHz}{ }^{1} \mathrm{H}$ NMR spectrum of compound 19

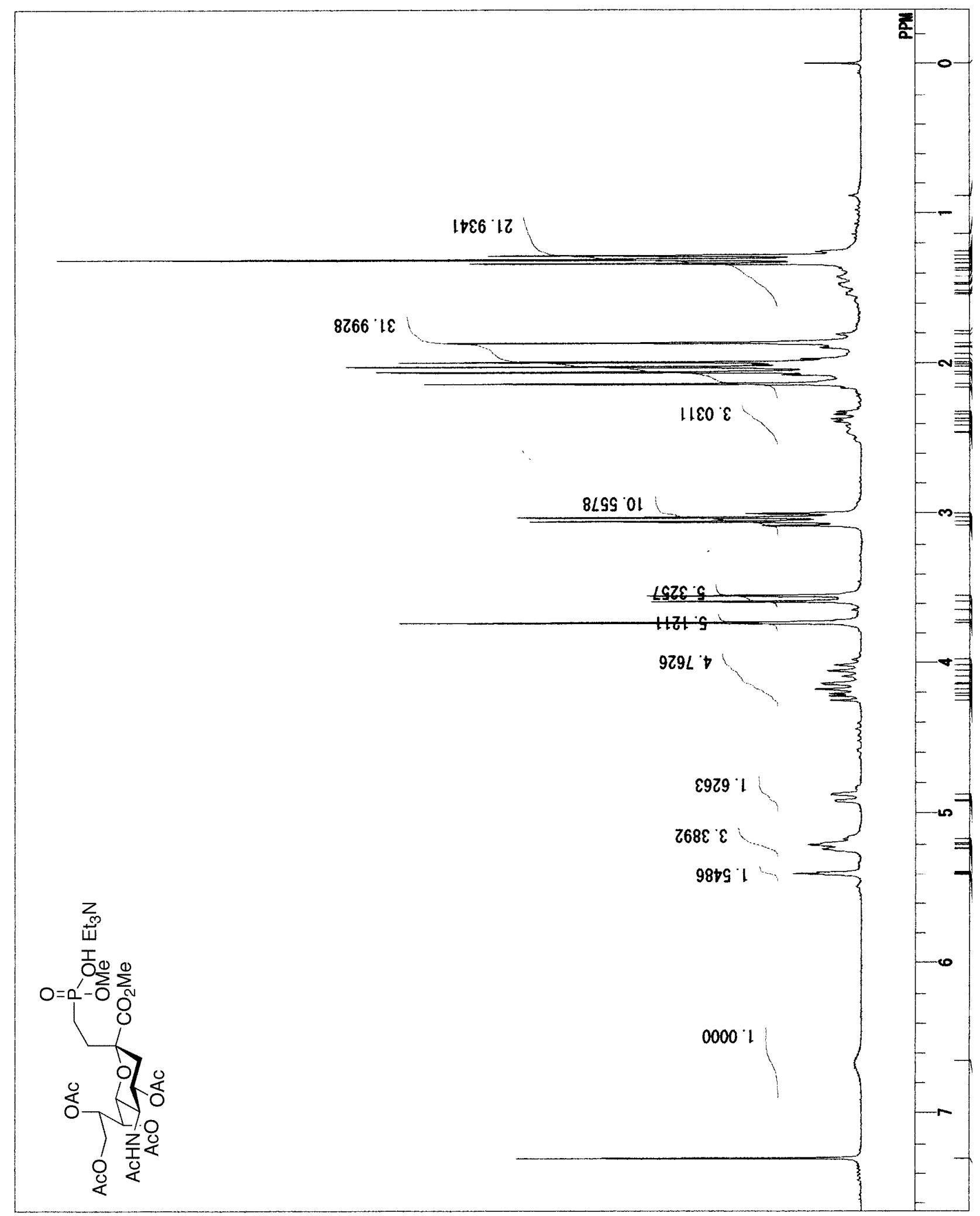




$$
7
$$


$270 \mathrm{MHz}{ }^{1} \mathrm{H}$ NMR spectrum of compound 21

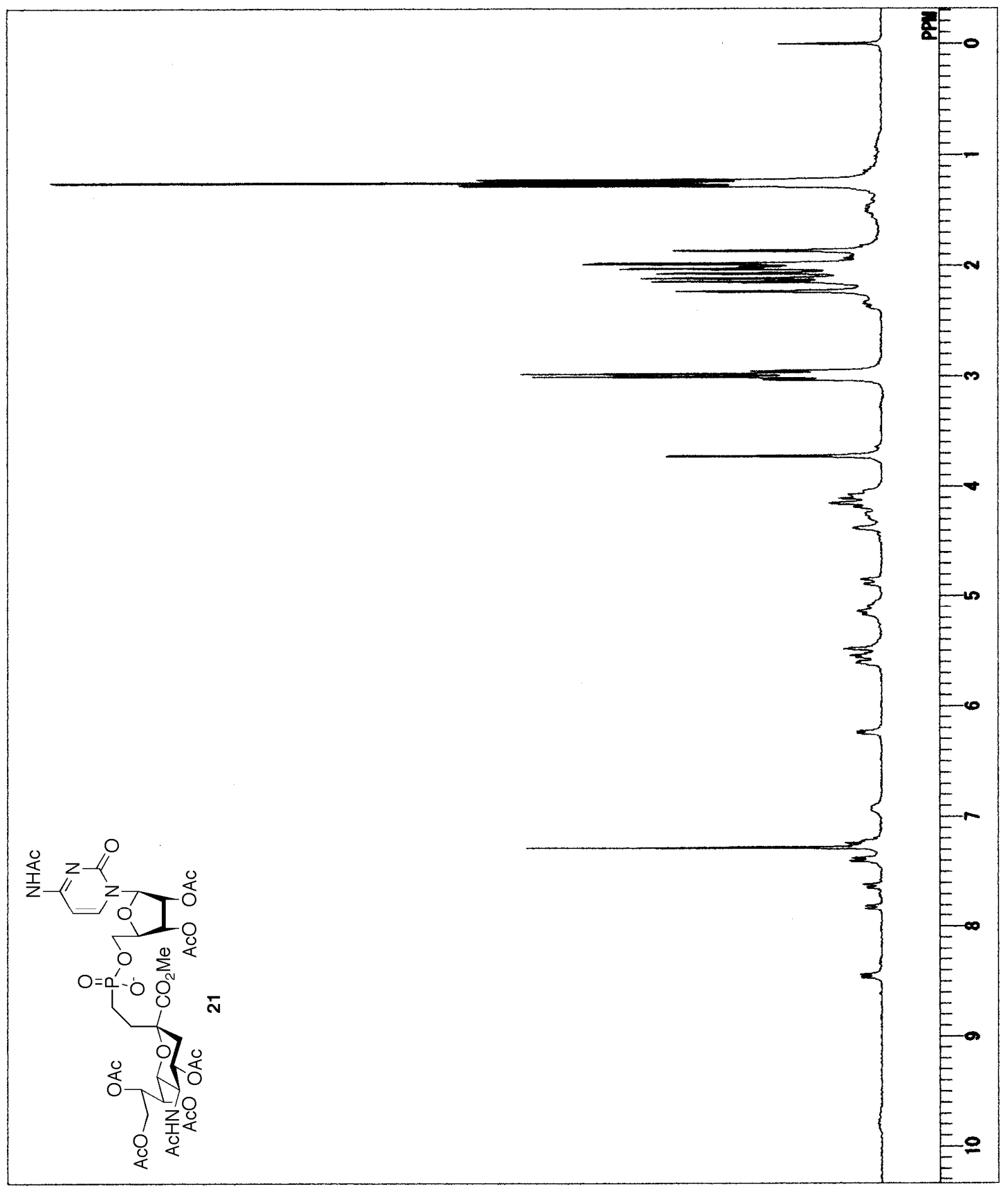


$67.8 \mathrm{MHz}{ }^{13} \mathrm{C}$ NMR spectrum of compound 21

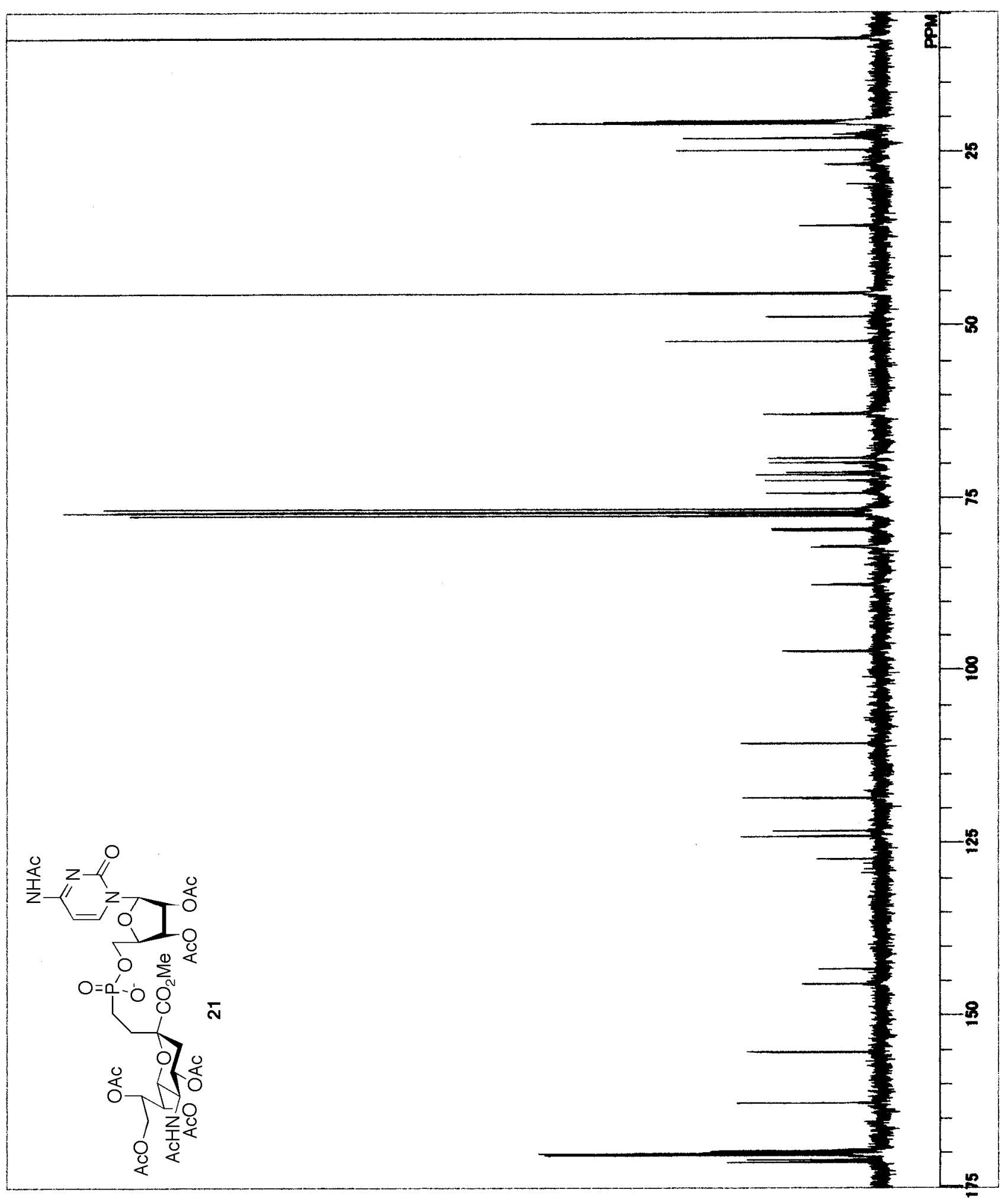


$400 \mathrm{MHz}{ }^{1} \mathrm{H}$ NMR spectrum of compound $\mathbf{4}$

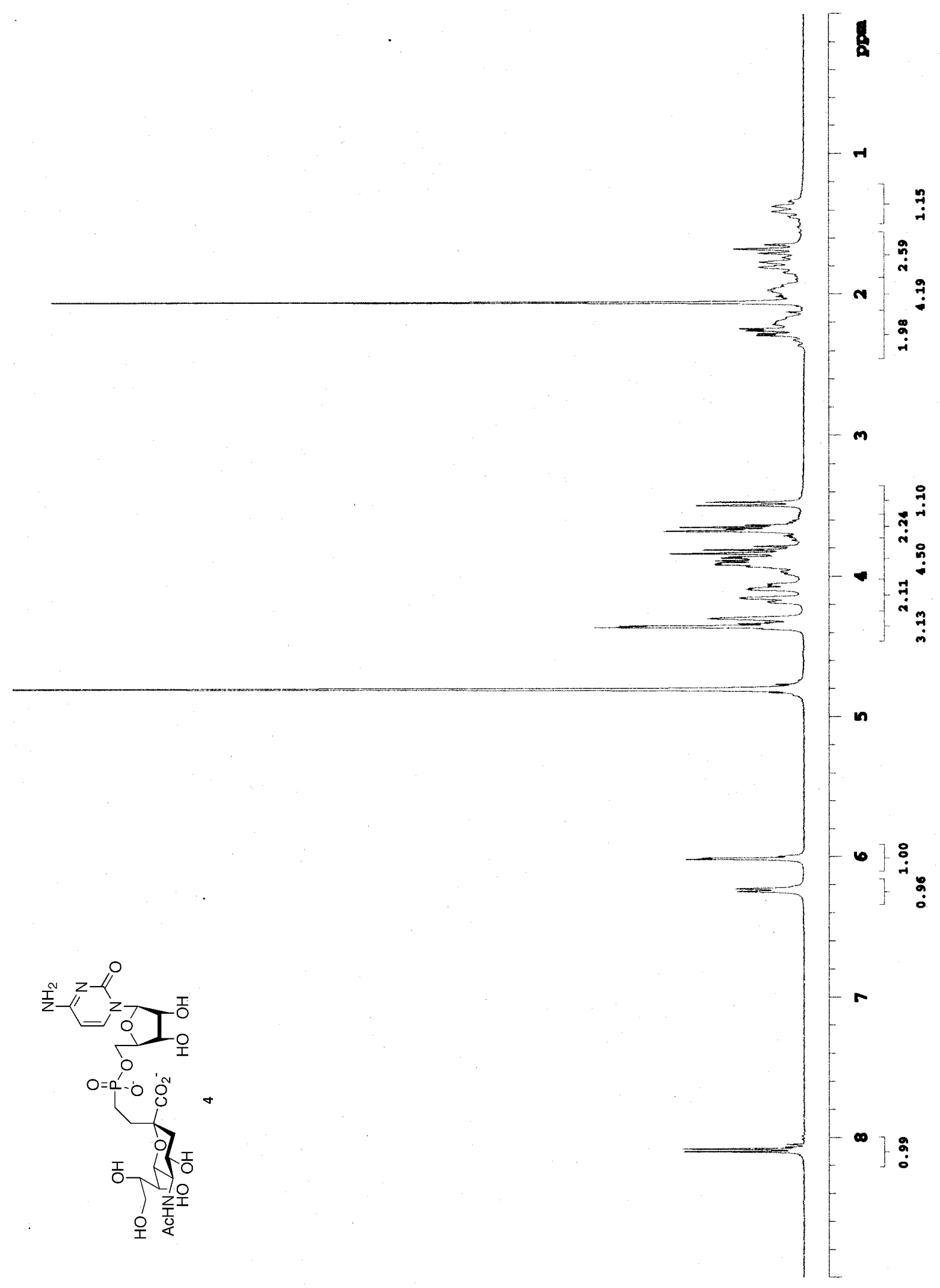


$100 \mathrm{MHz}{ }^{13} \mathrm{C}$ NMR spectrum of compound 4

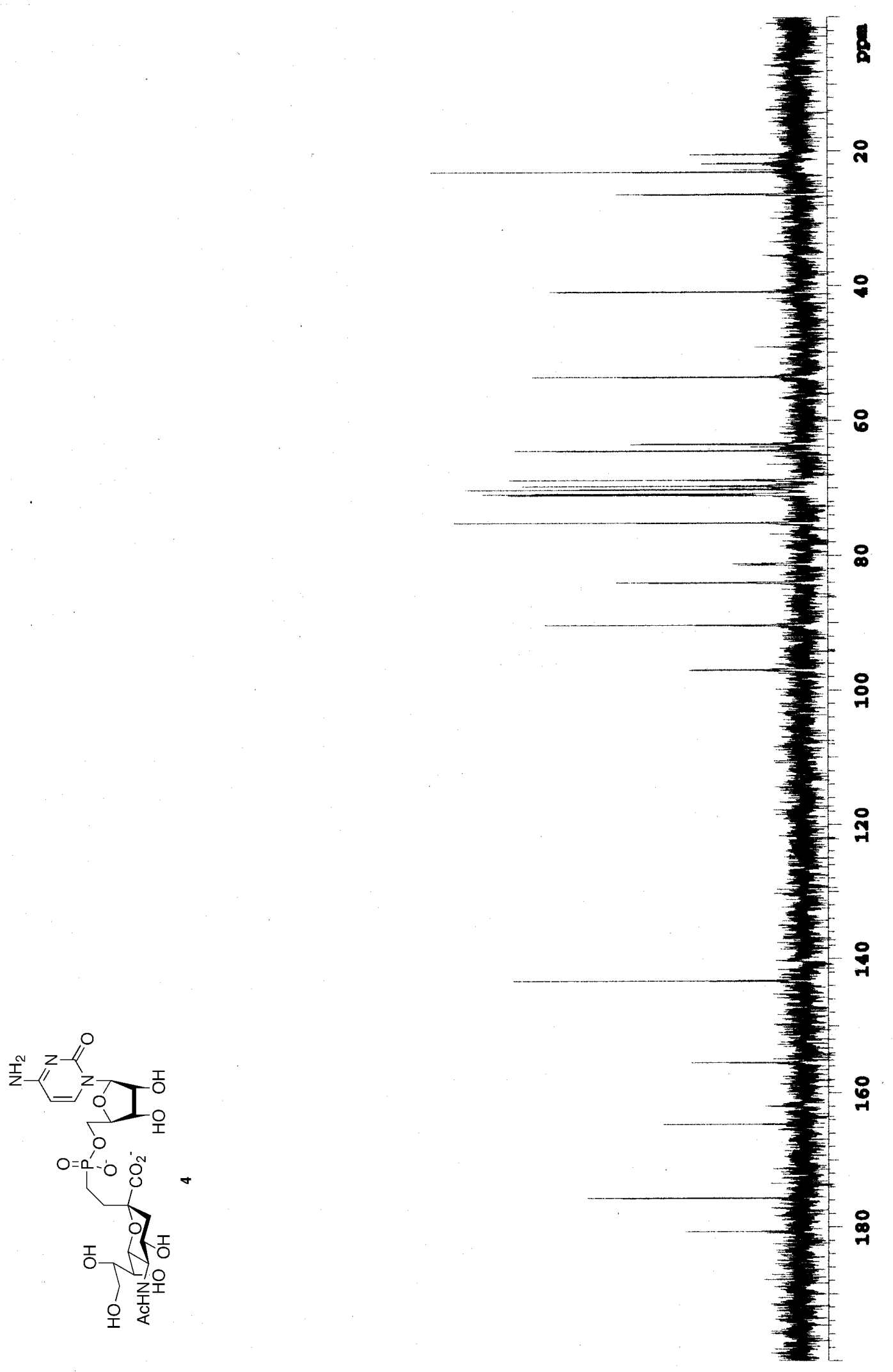


$400 \mathrm{MHz}{ }^{1} \mathrm{H}$ NMR spectrum of compound 5

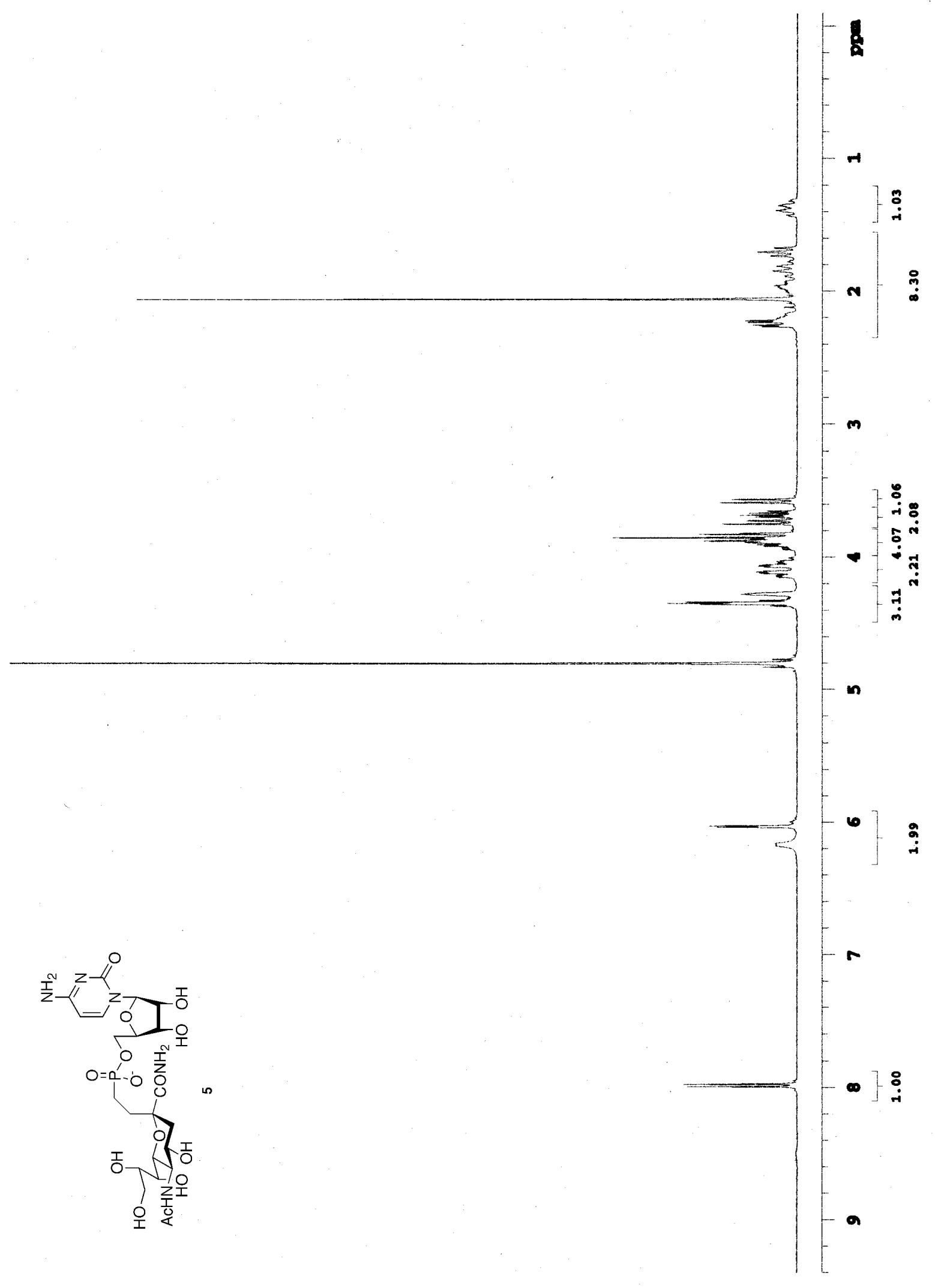


$100 \mathrm{MHz}{ }^{13} \mathrm{C}$ NMR spectrum of compound 5

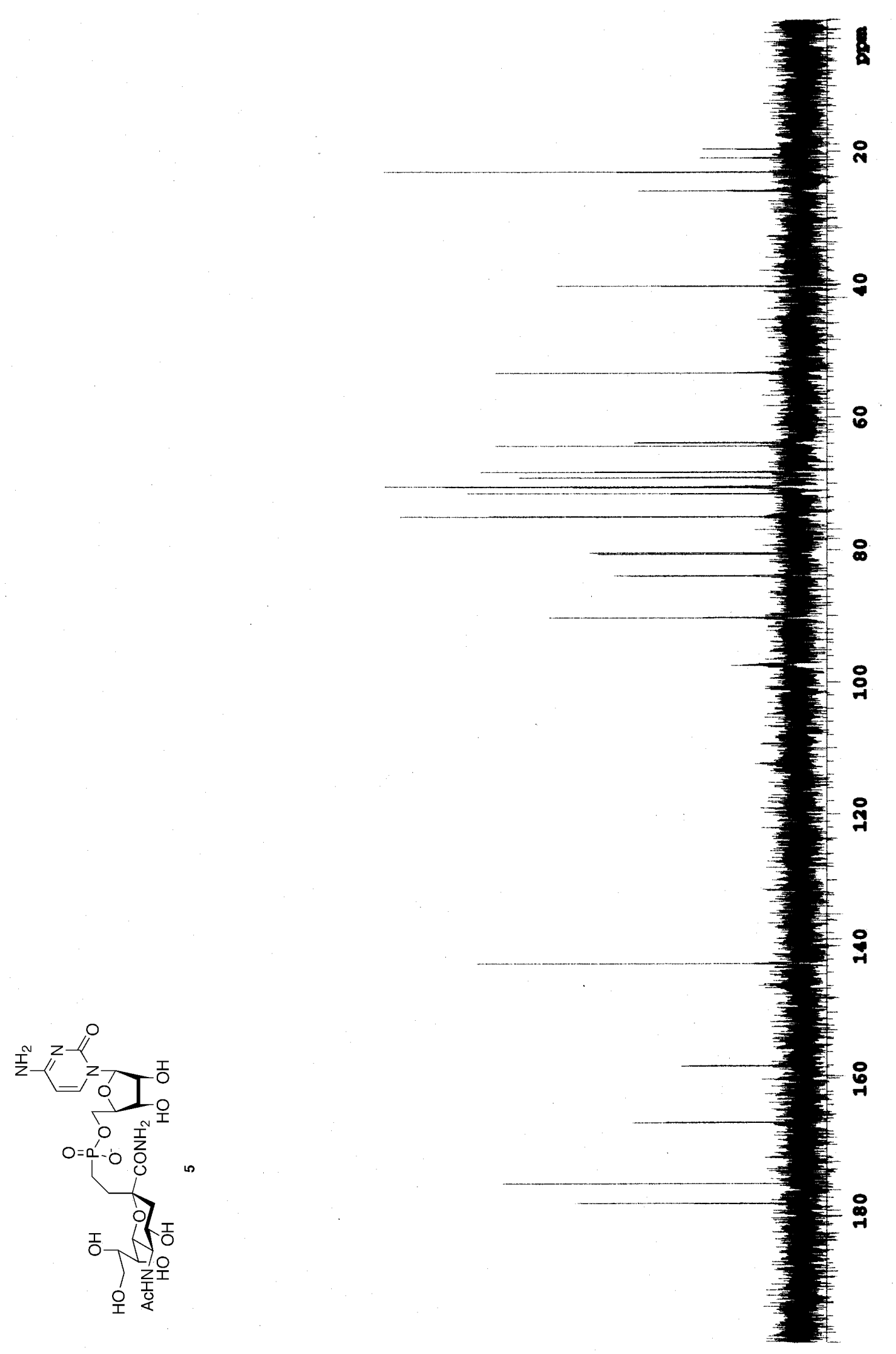


$270 \mathrm{MHz}{ }^{1} \mathrm{H}$ NMR spectrum of compound 22

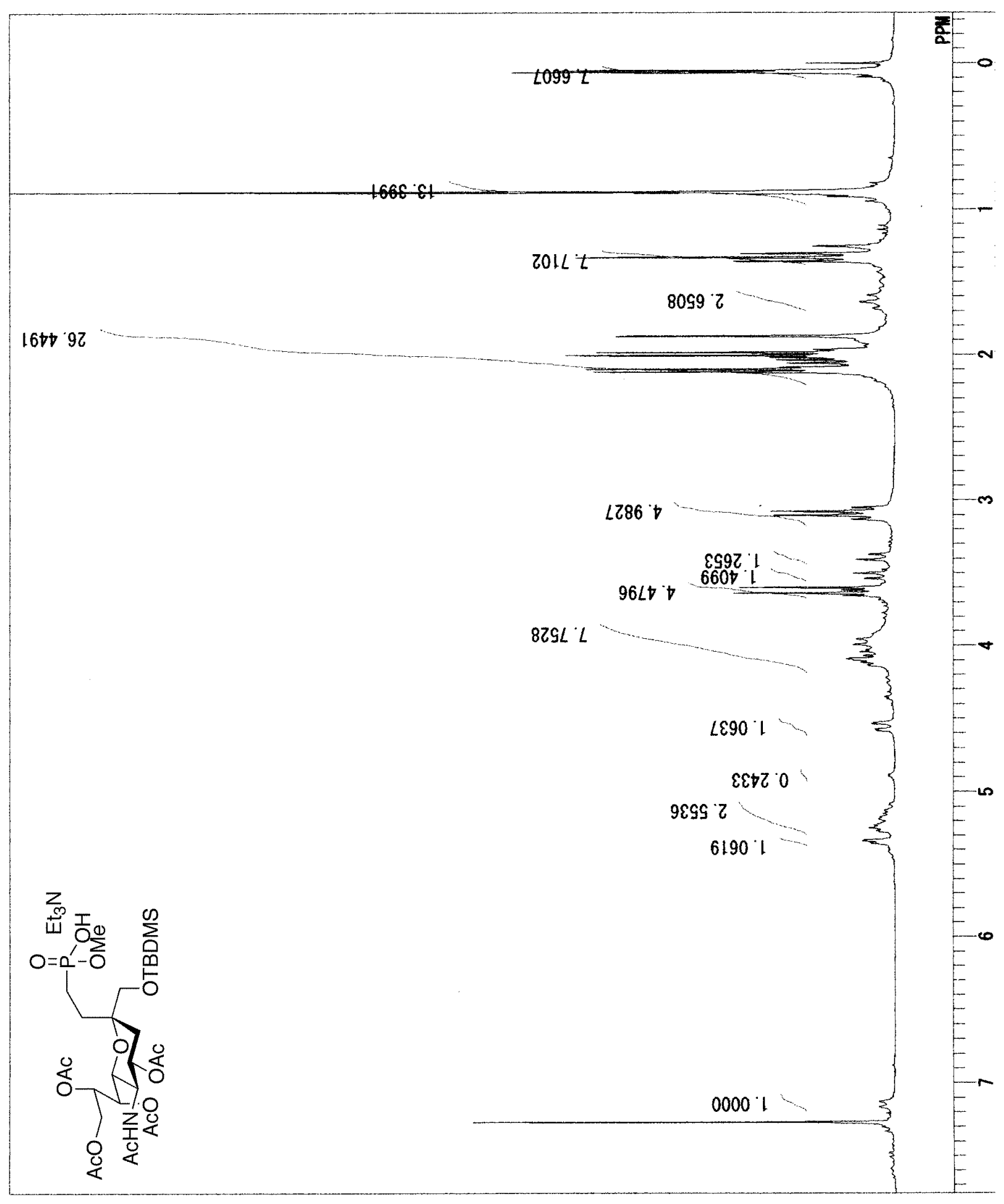


$67.8 \mathrm{MHz}{ }^{13} \mathrm{C}$ NMR spectrum of compound 22

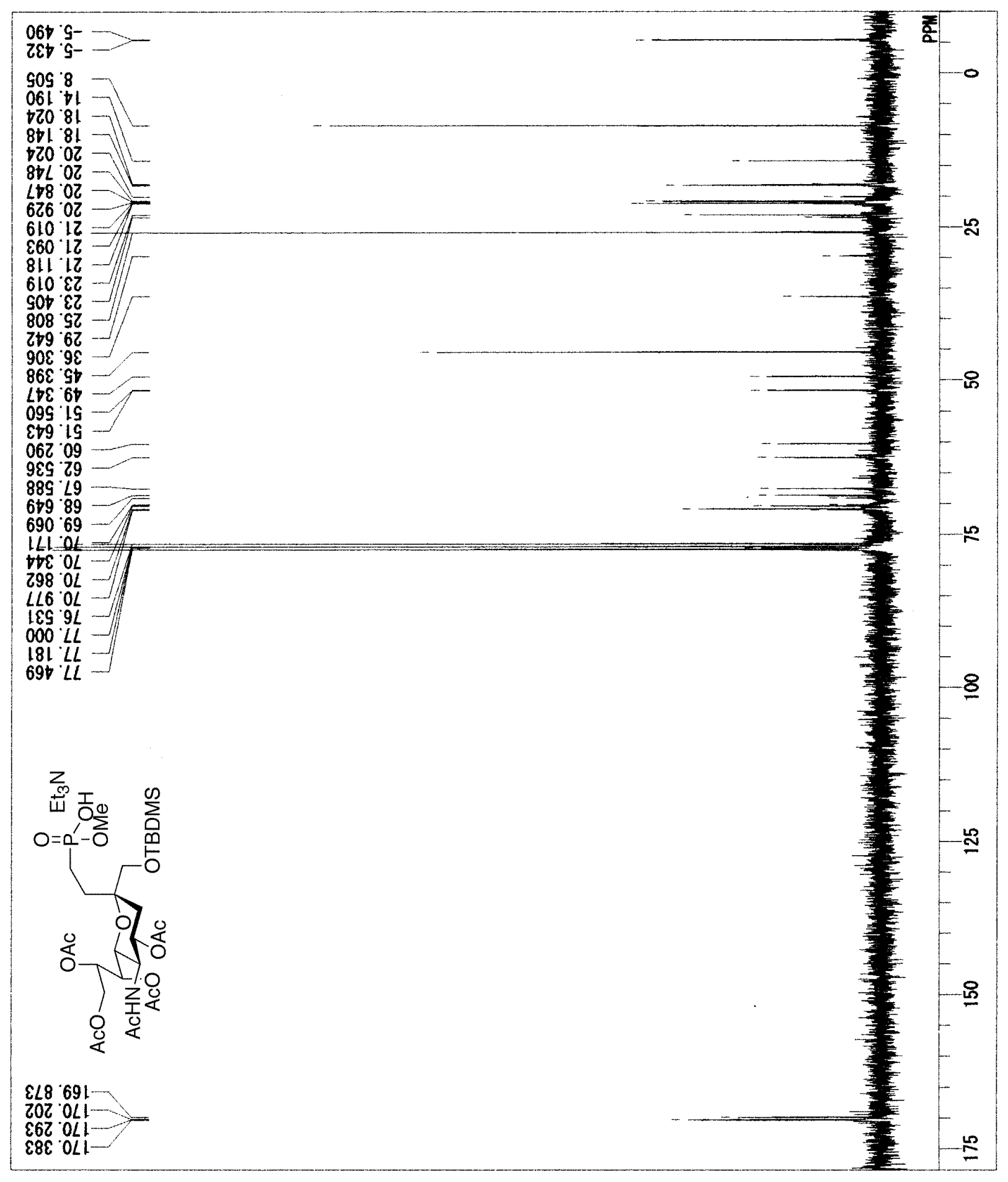


$270 \mathrm{MHz}{ }^{1} \mathrm{H}$ NMR spectrum of compound 23

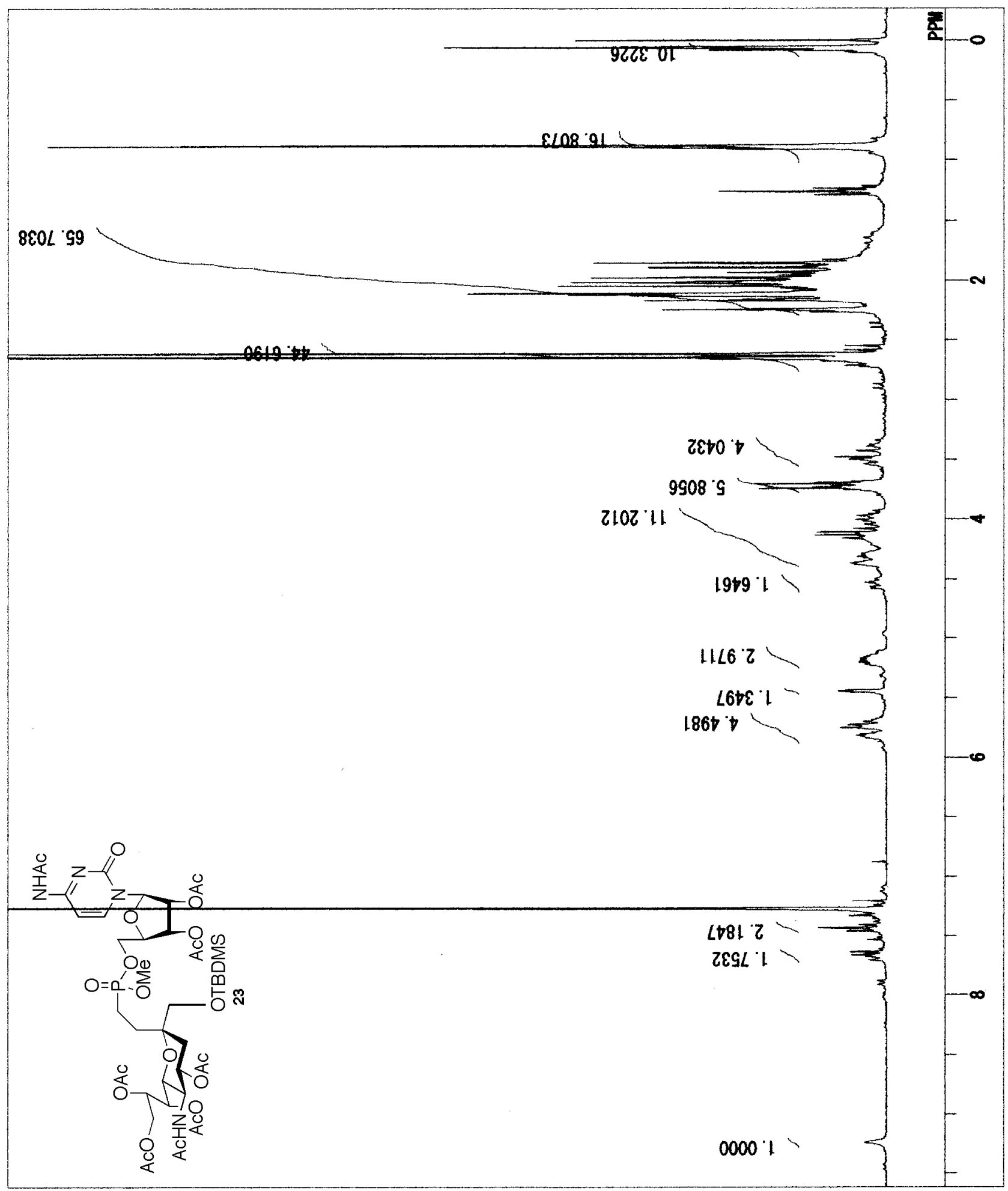


$400 \mathrm{MHz}{ }^{1} \mathrm{H}$ NMR spectrum of compound 6

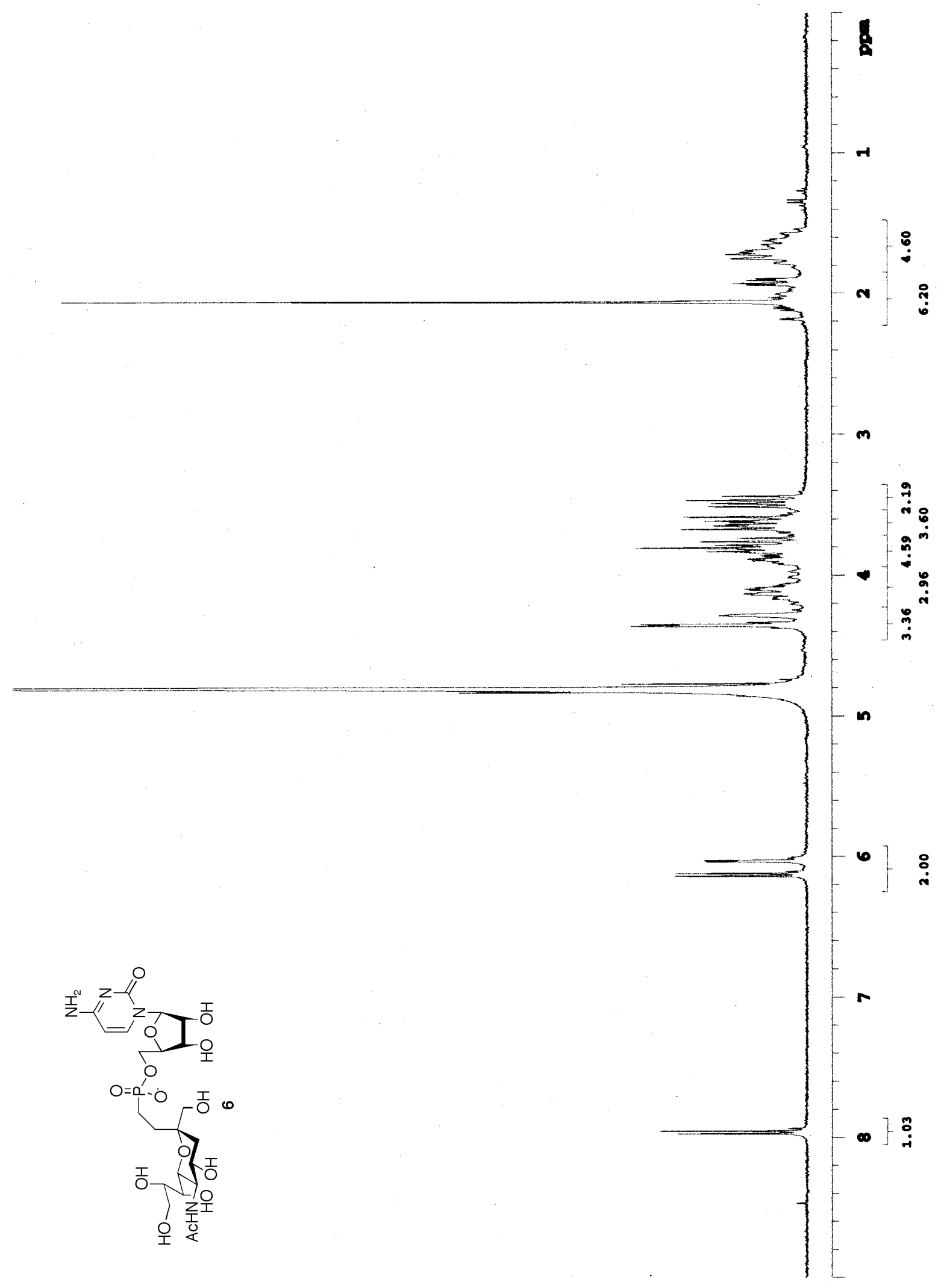


$100 \mathrm{MHz}{ }^{13} \mathrm{C}$ NMR spectrum of compound 6

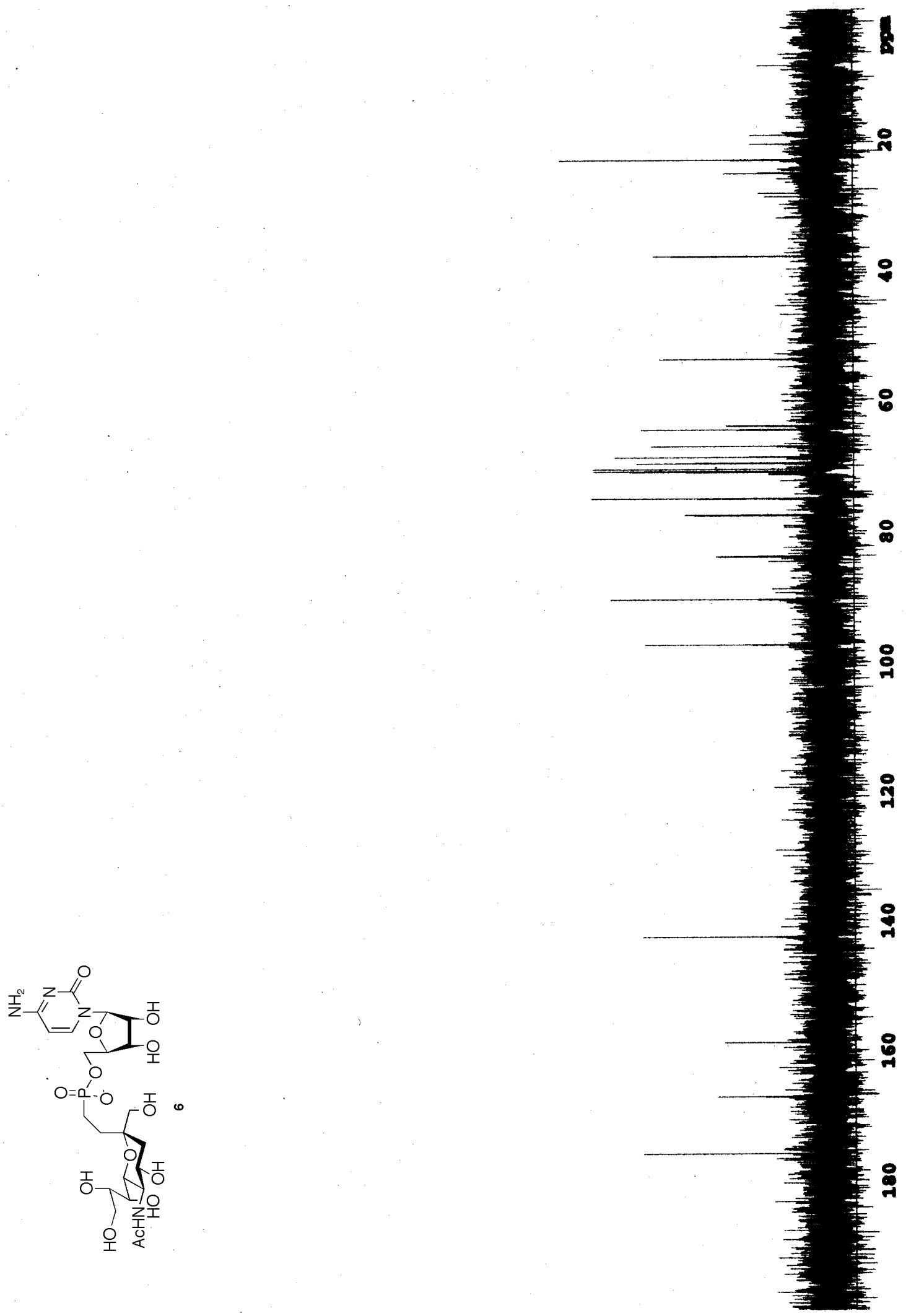


$270 \mathrm{MHz}{ }^{1} \mathrm{H}$ NMR of compound 24

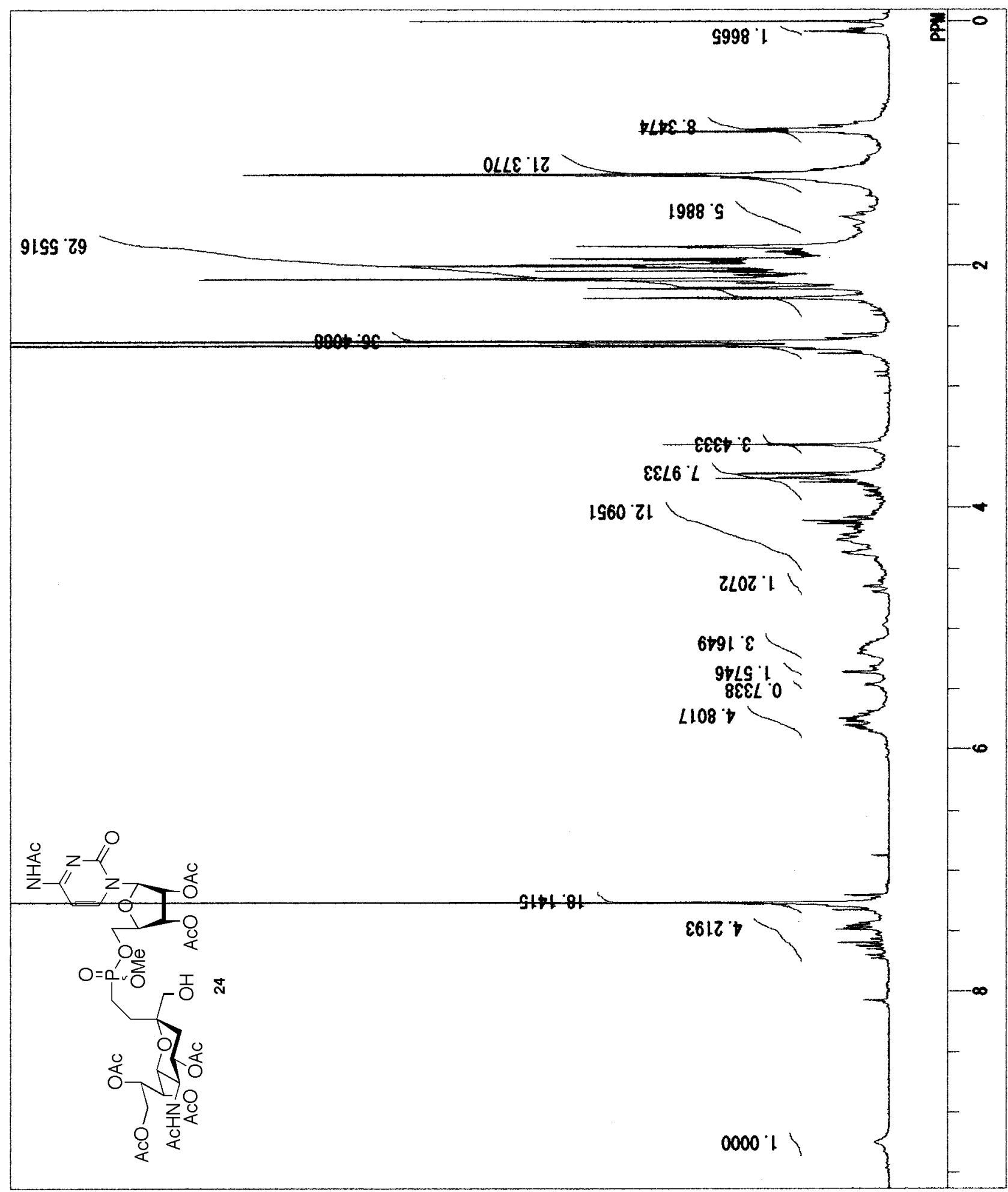


$400 \mathrm{MHz}{ }^{1} \mathrm{H}$ NMR spectrum of compound 7

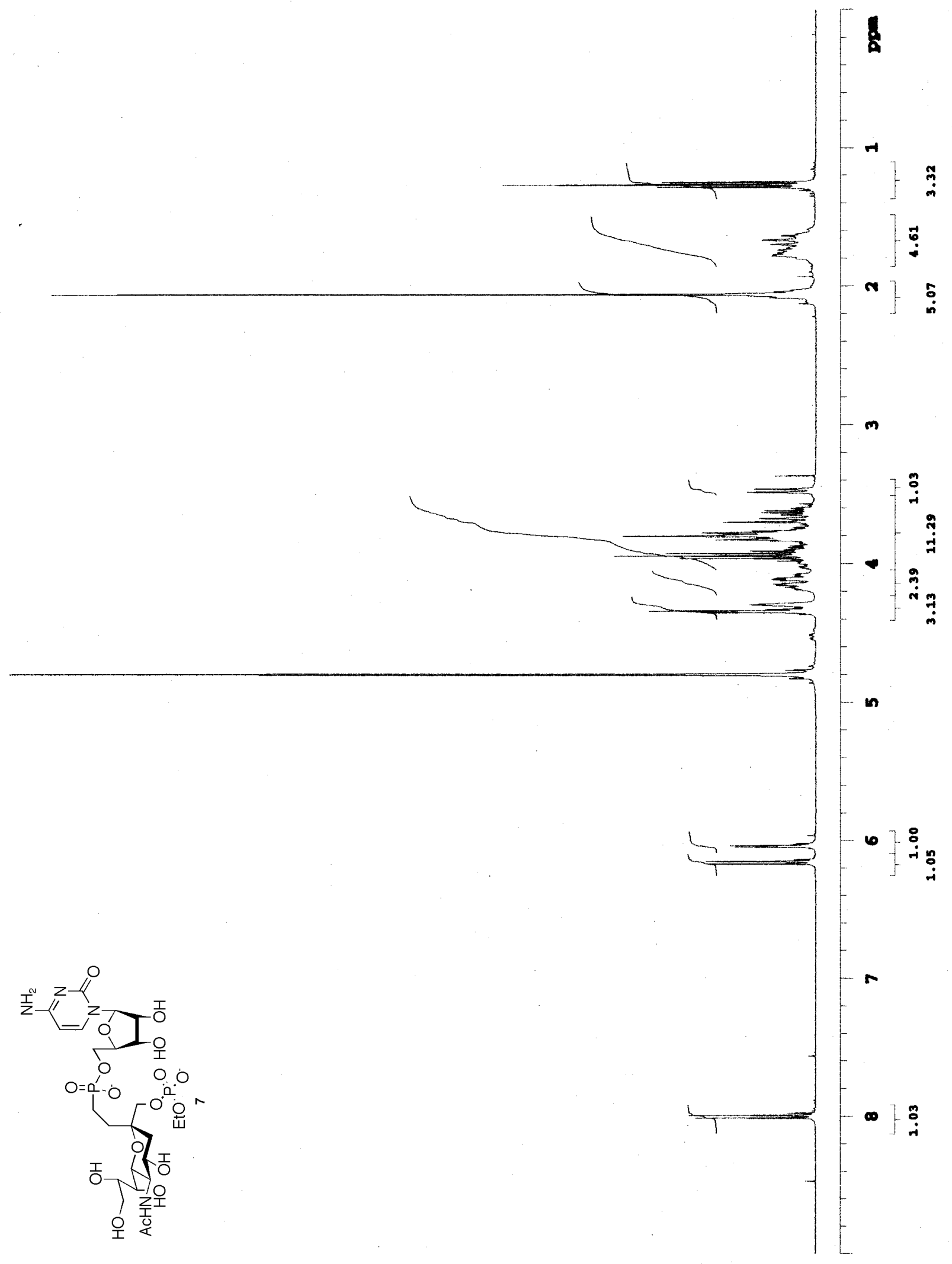


$100 \mathrm{MHz}{ }^{13} \mathrm{C}$ NMR spectrum of compound 7

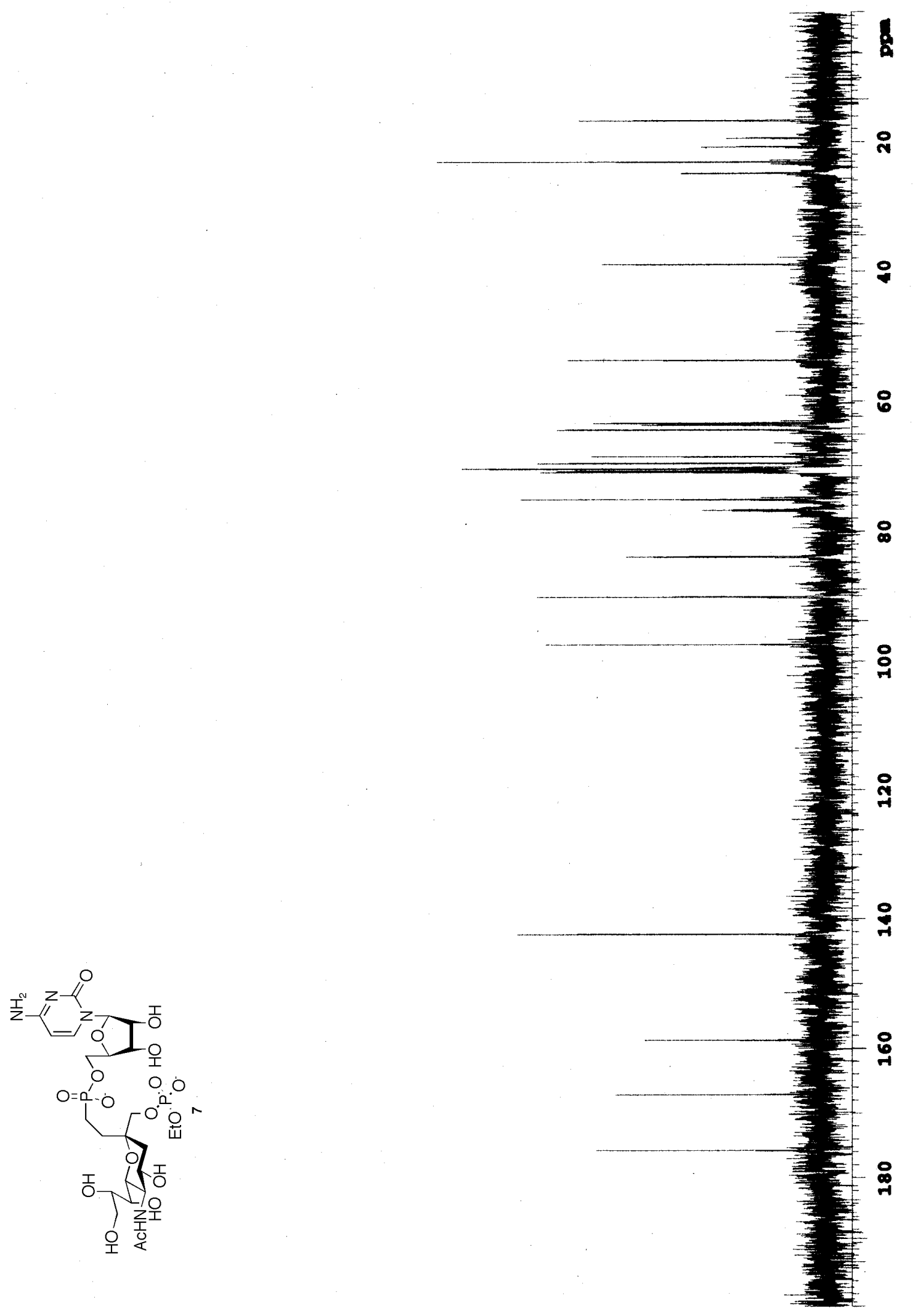


$270 \mathrm{MHz}{ }^{1} \mathrm{H}$ NMR of compound 26

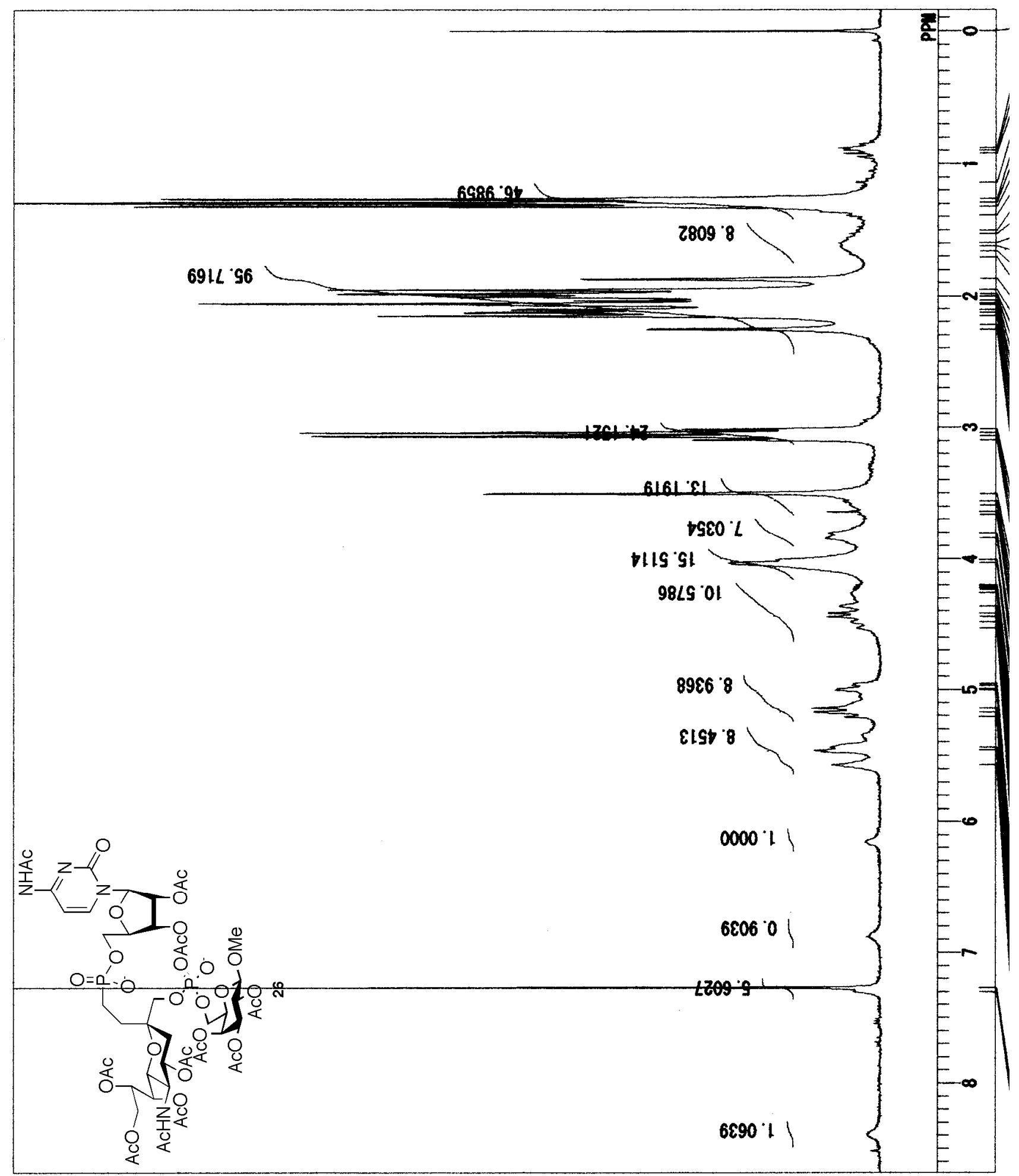


$400 \mathrm{MHz}{ }^{1} \mathrm{H}$ NMR spectrum of compound 8

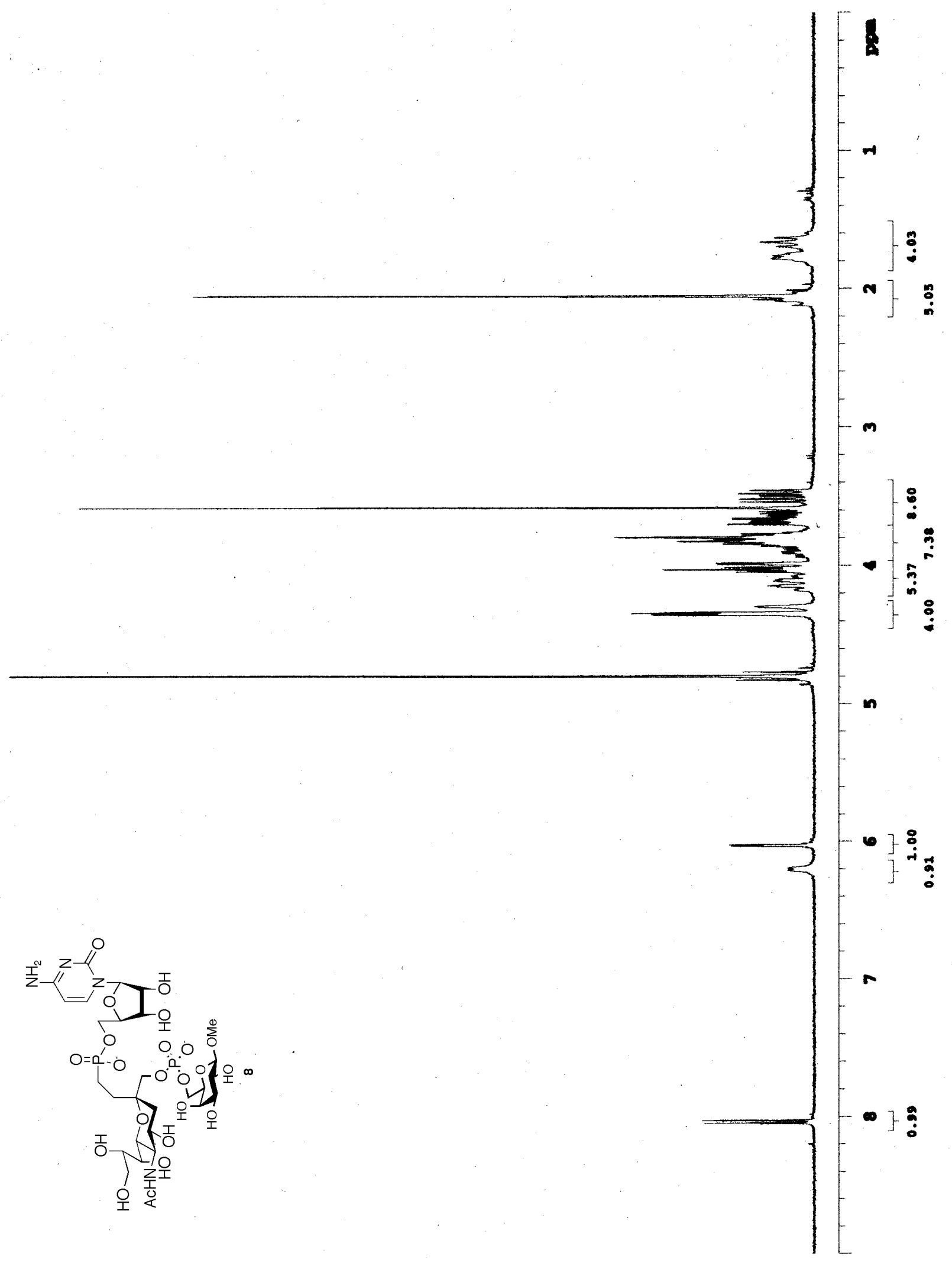


$100 \mathrm{MHz}{ }^{13} \mathrm{C}$ NMR spectrum of compound 8

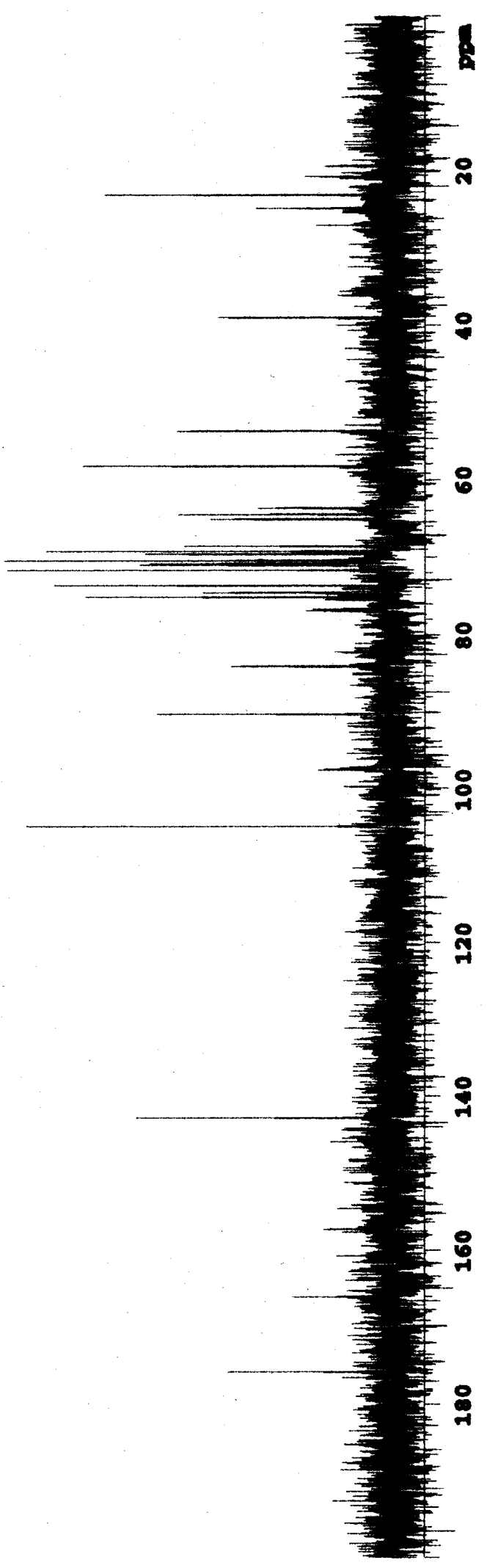

